\title{
ON NEUMANN TYPE PROBLEMS FOR NONLOCAL EQUATIONS SET IN A HALF SPACE
}

\author{
GUY BARLES, EMMANUEL CHASSEIGNE, CHRISTINE GEORGELIN, \\ AND ESPEN R. JAKOBSEN
}

\begin{abstract}
We study Neumann type boundary value problems for nonlocal equations related to Lévy type processes. Since these equations are nonlocal, Neumann type problems can be obtained in many ways, depending on the kind of "reflection" we impose on the outside jumps. To focus on the new phenomena and ideas, we consider different models of reflection and rather general nonsymmetric Lévy measures, but only simple linear equations in half-space domains. We derive the Neumann/reflection problems through a truncation procedure on the Lévy measure, and then we develop a viscosity solution theory which includes comparison, existence, and some regularity results. For problems involving fractional Laplace $(-\Delta)^{\frac{\alpha}{2}}$-like nonlocal operators, we prove that solutions of all our nonlocal Neumann problems converge as $\alpha \rightarrow, 2^{-}$ to the solution of a classical local Neumann problem. The reflection models we consider include cases where the underlying Lévy processes are reflected, projected, and/or censored when exiting the domain.
\end{abstract}

\section{INTRODUCTION}

In the probabilistic approach to elliptic and parabolic partial differential equations, it is well known that Neumann type boundary conditions are associated to stochastic processes being reflected on the boundary. We refer the reader to the book of Freidlin [14] for an introduction and to Lions and Sznitman [23] for general results. A key result in this direction is roughly speaking the following: for a PDE with Neumann or oblique boundary conditions, there is a unique underlying reflection process, and any consistent approximation will converge to it in the limit (see [23] and Barles and Lions [7]). At least in the case of normal reflections, this result is strongly connected to the study of the Skorohod problem and relies on the underlying stochastic processes being continuous.

The starting point of this article is to address the same question for jump processes related to partial integrodifferential equations (PIDEs for short). What is a reflection for such processes, and is a PIDE with Neumann boundary conditions naturally connected to a reflection process? It turns out that the situation is more complicated in this setting, at least the questions have to be formulated in a different way. In this article we address these questions through an analytical PIDE

Received by the editors December 2, 2011 and, in revised form, January 17, 2013.

2010 Mathematics Subject Classification. Primary 35R09; Secondary 45K05, 35D40.

Key words and phrases. Nonlocal equations, Neumann boundary conditions, jumps, Lévy measure, reflection, viscosity solutions.

The fourth author was supported by the Research Council of Norway through the project "DIMMA". 
approach where we keep in mind the idea of having a reflecting process but without defining it precisely or even proving its existence.

For jump processes which are discontinuous and may exit a domain without first hitting its boundary, there are many ways to define a "reflection" or a "reflecting process". Also, because of the way the PIDE and the process are related, defining a reflection on the boundary will change the equation inside the domain. This is a new nonlocal phenomenon which is not encountered in the case of continuous processes and PDEs.

PIDE with Neumann type boundary condition. In order to simplify the presentation and focus on the main new ideas and phenomenas, we consider several different models of reflections and rather general nonsymmetric Lévy measures, but only for problems involving linear equations set in simple domains. The cases we will consider already have interesting features and difficulties. We consider the half space $\Omega:=\left\{\left(x_{1}, \ldots, x_{N}\right)=\left(x^{\prime}, x_{N}\right) \in \mathbb{R}^{N}: x_{N}>0\right\}$ and simple linear Neumann type problems that we write as

$$
\begin{cases}u(x)-I[u](x)-f(x)=0 & \text { in } \quad \Omega, \\ -\frac{\partial u}{\partial x_{N}}=0 & \text { in } \quad \partial \Omega\end{cases}
$$

or

$$
\begin{cases}F(x, u, I[u])=0 & \text { in } \quad \Omega, \\ -\frac{\partial u}{\partial x_{N}}=0 & \text { in } \quad \partial \Omega,\end{cases}
$$

where $F(x, r, l)=r-l-f(x)$ and

$$
I[u](x)=\lim _{\delta \rightarrow 0^{+}} \int_{|z|>\delta}[u(x+\eta(x, z))-u(x)] d \mu(z)
$$

We will assume that $f \in C_{b}(\bar{\Omega})$, i.e. $f$ is bounded and continuous, that $\mu$ is a nonnegative Radon measure satisfying

$$
\int|z|^{2} \wedge 1 d \mu(z)<\infty
$$

and that

$$
x+\eta(x, z) \in \bar{\Omega} \text { for all } x \in \bar{\Omega}, \eta(x, z)=z \text { if } x+z \in \bar{\Omega} .
$$

Note that $I[u]$ is a principal value (P.V.) integral and that (1.2) is the most general integrability assumption satisfied by Lévy measures [1. When $\eta(x, z) \equiv z$, then $I[u]$ is the generator of a stochastic process which can jump from $x \in \bar{\Omega}$ to $x+z$ with a certain intensity; see e.g. [1,12,15. Assumption (1.3) is a type of reflection condition preventing the jump process from leaving the domain: nothing happens and $\eta(x, z)=z$ if $x+z \in \bar{\Omega}$, while if $x+z \notin \bar{\Omega}$, then a "reflection" is performed in order to move the particle back to a point $P(x, z)=x+\eta(x, z)$ inside $\bar{\Omega}$. Note that we have to check at some point that the reflection is consistent with a Neumann boundary condition. In this paper the boundary value problem (1.1) will be interpreted in the sense of viscosity solutions. 
The main examples of $\eta$ are the following model cases, where we use the notation $x=\left(x^{\prime}, x_{N}\right) \in \mathbb{R}^{N-1} \times \mathbb{R}_{+}, \eta(x, z)=\left(\eta(x, z)^{\prime}, \eta(x, z)_{N}\right)$, etc.:
(a) $\eta(x, z)= \begin{cases}z & \text { if } x_{N}+z_{N} \geq 0, \\ 0 & \text { if not }\end{cases}$
[censored],
(b) $\eta(x, z)= \begin{cases}z & \text { if } x_{N}+z_{N} \geq 0 \\ z \frac{x_{N}}{\left|z_{N}\right|} & \text { if not }\end{cases}$
[fleas on the window],
(c) $\eta(x, z)= \begin{cases}z & \text { if } x_{N}+z_{N} \geq 0, \\ \left(z^{\prime},-x_{N}\right) & \text { if not }\end{cases}$
[normal projection],
(d) $\eta(x, z)= \begin{cases}z & \text { if } x_{N}+z_{N} \geq 0 \\ \left(z^{\prime},-2 x_{N}-z_{N}\right) & \text { if not }\end{cases}$
[mirror reflection]

for all $x \in \Omega$ and $z \neq 0$. The different reflections are depicted in Figure 1. We will discuss later whether the naively proposed "reflections" are realized by a concrete Markov process, i.e. if they correspond to the generator of such a process.

Main results. From an analytical (PIDE) point of view, the first step is to give a sense to problem (1.1) and relate it to a homogeneous Neumann boundary value problem. This is done in Sections 2 and 3. The first part is classical: to take into account singular Lévy measures, we write the integral operator with a compensator term, using also the principal value. Here classical arguments in viscosity solution theory are used; see e.g. [6] and the references therein. Viscosity solutions' theory also provides a suitable definition of "generalized" boundary conditions (cf. the user's guide [13]) which is used, here, for the Neumann boundary conditions.

If $\mu$ is a bounded measure, then problem (1.1) can be solved easily without caring much about the Neumann boundary condition. Moreover, the solutions will be uniformly bounded by $\|f\|_{\infty}$. From the modelling point of view, (1.1) carries the information that the particles of the system remain in $\bar{\Omega}$ since they can only jump inside $\bar{\Omega}$. This mass conservation is an other way to understand that we are dealing with a (homogeneous) Neumann type of boundary condition.

When $\mu$ is a singular measure, we can approximate it by a sequence of bounded measures $\left(\mu_{n}\right)_{n}$, consider the associated (uniformly bounded) solutions $\left(u_{n}\right)_{n}$, and wonder what the limiting problem is. This is the way we choose to make sense of both the definition of problem (1.1) and the associated notion of (viscosity) solutions. We point out here that the Neumann boundary condition is active only if the measure is singular enough. Otherwise the equation will hold up to the boundary, as in the case of reflecting $\alpha$-stable process with $\alpha<1$ where the process will never reach the boundary 9 .

A natural next step is to prove uniqueness results for the above equations in the different cases. Different proofs are given depending on the singularity of the measure and the structure of the "reflection" mechanism at the boundary. They are given in Sections 4- 6. The first case we treat is when the jump function $\eta$ enjoys a contraction property in the normal direction. This covers all the noncensored models (b)-(d). Had we had a contraction in all directions, then the usual viscosity solution doubling of variables argument would work. Here we have to modify that argument to take into account the special role of the normal direction. 


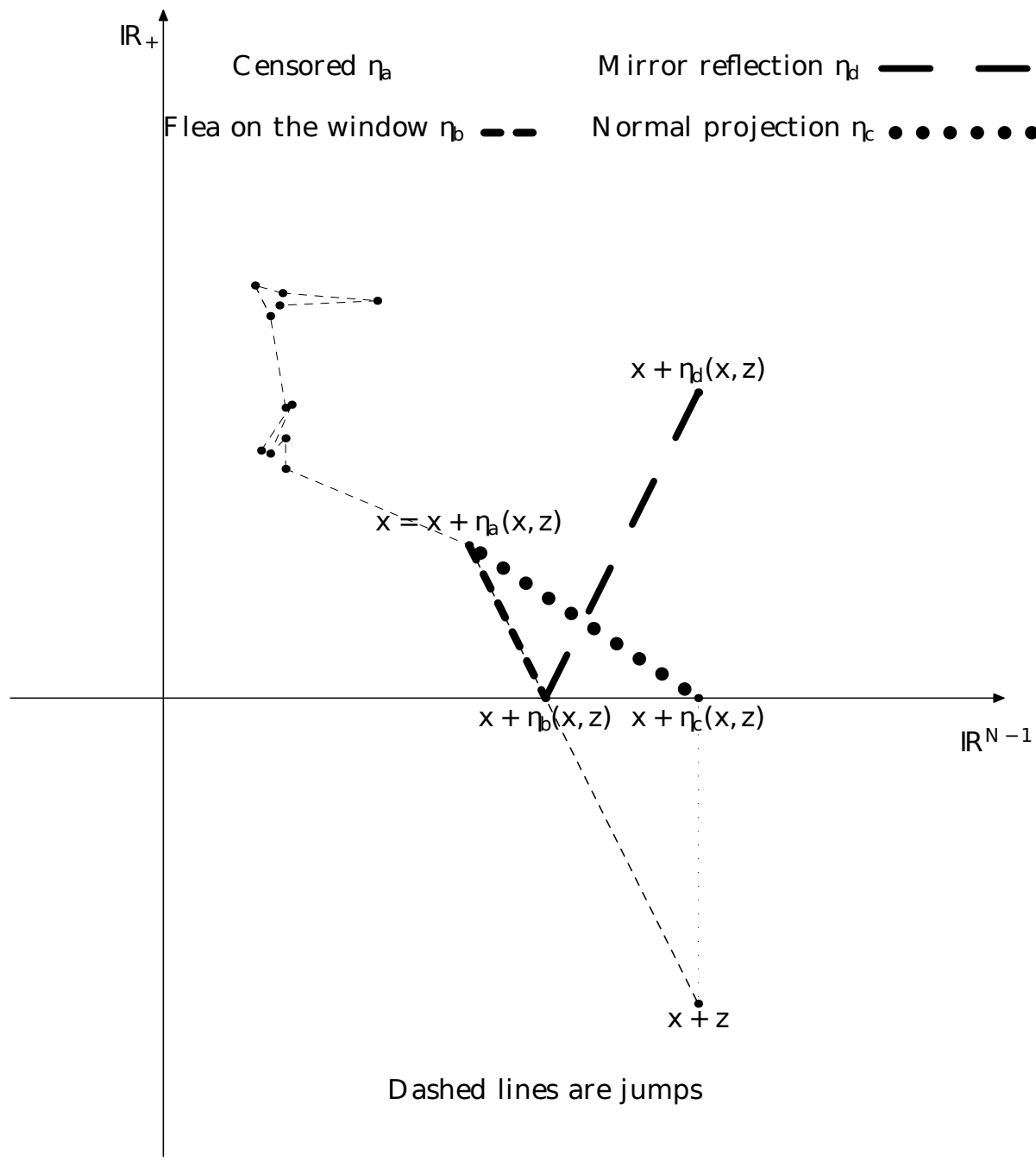

Figure 1

The second case we consider is the censored case (a) when the singularity of the measure is not too strong. By this we mean typically a stable process with Lévy measure with density $\frac{d \mu}{d z} \sim \frac{1}{|z|^{N+\alpha}}$ for $\alpha \in(0,1)$. We construct an approximate subsolution which blows up at the boundary, and this allows us to derive a comparison result by a penalization procedure. Such a construction is related to the fact that the process does not reach the boundary in this case; see e.g. 9].

The last case is the censored case (a) when the singularity is strong, i.e. when $\alpha \in[1,2)$. This case requires much more work because no blow-up subsolutions exist here. In fact, when $\alpha \in[1,2)$, the censored process does reach the boundary (see e.g. [9]). We first prove that the Neumann boundary condition is already encoded in the equation under the additional assumption that the solution is $\beta$-Hölder continuous at the boundary for some $\beta>\alpha-1$. Then we prove a comparison result for sub/supersolutions with this Hölder regularity at the boundary. The proof is 
then similar to the proof in the $\alpha<1$ case, except that the special subsolutions in this case are bounded and only penalize the boundary when the sub/supersolutions are Hölder continuous there with $\beta>\alpha-1$. Finally, we construct solutions in this class. In dimension $N=1$, we use and prove that any bounded uniformly continuous solution is Hölder continuous provided $\mu$ satisfies some additional integrability condition. In higher dimensions, we use and prove a similar result under additional regularity assumptions on $f$ in the tangential directions.

Finally, in Section 7 we show that our models are consistent with the local Neumann problem: All the proposed nonlocal models converge to the same local Neumann problem when the Lévy measure approches the "local" case $\alpha=2$. More precisely, we consider Lévy measures $\mu_{\alpha}$ with densities $(2-\alpha) \frac{g(z)}{|z|^{N+\alpha}}$, where $g$ is a nonnegative bounded function which is $C^{1}$ in a neighborhood of 0 and satisfies $g(0)>0$. In this case we prove that whatever nonlocal Neumann model we use, the solutions $u_{\alpha}$ converge as $\alpha \rightarrow 2$ to the unique viscosity solution of the same limit problem, namely

$$
\left\{\begin{array}{cl}
-a \Delta u-b \cdot D u+u=f & \text { in } \Omega \\
\frac{\partial u}{\partial \mathbf{n}}=0 & \text { in } \partial \Omega
\end{array}\right.
$$

where $a:=g(0) \frac{\left|S^{N-1}\right|}{N}$ and $b:=D g(0) \frac{\left|S^{N-1}\right|}{N}$. For problems without boundary conditions, such asymptotic results have been known for a long time; we refer e.g. to [8,10] and the references therein for more details.

Related work. One of the first papers on this subject was by Menaldi and Robin [21. In that paper stochastic differential equations for reflection problems were solved in the case of diffusion processes with inside jumps, i.e. when there are no jumps outside the domain. They used the method of "penalization on the domain" inspired by Lions, Menaldi and Sznitman [24].

In model (a) (the censored case), any outside jump of the underlying process is cancelled (censored) and the process is restarted (resurrected) at the origin of that jump. We refer to e.g. 99, 15, 18, 20, 22 for more details on such processes. The process can be constructed using the Ikeda-Nagasawa-Watanabe piecing together procedure [9, 15, 22, as Hunt processes associated to some Dirichlet forms [9, 18, or via the Feynman-Kac transform involving the killing measure 9. In particular, the underlying processes in this paper are related to the censored stable processes of Bogdan et al. [9] and the reflected $\alpha$-stable process of Guan and Ma [18. Approximations by truncated measures have been considered in the work of Kim [20, on the weak convergence of censored and reflected stable processes. But note that we essentially only study the generators and not yet the processes themselves. On the technical side, we use viscosity solution methods, while [9, 18, 20 use the theory of Dirichlet forms and potential theory. Our assumptions are also different, e.g. with our arguments we treat more general measures and we have the potential to treat nonlinear problems, while [9, 18, treat much more general domains.

Let us also mention that the "natural" Neumann boundary condition for the reflected $\alpha$-stable process of Guan and Ma [18, is different from the one we consider here. They show that the boundary condition arising through the variational 
formulation and Green type formulas is

$$
\lim _{t \rightarrow 0} t^{2-\alpha} \frac{\partial u}{\partial x_{N}}\left(x+t e_{N}\right)=0 .
$$

This formula allows the normal derivative $\frac{\partial u}{\partial x_{N}}$ to explode less rapidly than $\left|x_{N}\right|^{\alpha-2}$, and it is consistent (after one integration) with our assumption (6.2) in Theorem6.1 (the censored case). Formula (1.5) also justifies the use of boundary conditions in the viscosity sense since $\frac{\partial u}{\partial x_{N}}$ is not necessarily equal to 0 on the boundary for $\alpha<2$.

In model (b) outside jumps are stopped where the jump path hits the boundary, and then the process is restarted there. Model (c) is close to the approach of LionsSznitman in 23], and here outside jumps are immediately projected to the boundary along the normal direction. This type of model will be thoroughly investigated in the forthcoming paper [5] by three of the authors, but this time in the setting of fully nonlinear equations set in general domains. Note that models (b) and (c) coincide in one dimension, i.e. when $N=1$. Finally, in model (d), outside jumps are mirror reflected at the boundary. This is a natural way to define a "reflection", but the model may be problematic to set up and work with in general domains due to the possibility of multiple reflections.

To the best of our knowledge, processes with generators of the form (b)-(d) have not been considered before. For example, the works of Stroock [29] and Taira [30,31] seem not to cover our cases because their integrodifferential operators involve measures and jump vectors $\eta$ that are more regular than ours. Moreover in these works, it is the measure and not $\eta$ that prevents the process to jump outside $\Omega$.

In the case of symmetric $\alpha$-stable processes (a subordinated Brownian motion), our formulation follows after a "reflection" on the boundary. Such processes can be constructed from a Brownian motion by first subordinating the process and then reflecting it. Another possible way to construct a "reflected" process in this case would be to reflect the Brownian motion first and then subordinate the reflected process. These two approaches are well understood for Dirichlet type of problems involving subordinated killed and killed subordinated process; cf. e.g. [27.

Another approach that is related to reflected subordinated processes is described e.g. in Hsu [19] (but see also 25]), where pure jump processes such as Lévy processes are connected via the Dirichlet-Neumann operator to the trace at the boundary of a reflected brownian motion in a space with an extra dimension $\Omega \times \mathbb{R}_{+}$. An analytic PIDE version of this approach was introduced by Caffarelli and Silvestre in [10] in order to obtain Harnack inequalities for solutions of integrodifferential equations, and since then these ideas have been used by many authors.

Finally we mention the more classical work of Garroni and Menaldi [16], where a large class of uniformly elliptic integrodifferential equations are considered. There are two main differences with our work: (i) In [16] the principle part of all equations is a local nondegenerate 2 nd order term. This allows the nonlocal terms to be controlled by local terms (the solution and its 1 st and 2 nd derivatives) via interpolation inequalities, and the local $W^{2, p}$ and $C^{2, \alpha}$ theories can therefore be extended to this nonlocal case. In our paper, it is the nonlocal terms that are the principal terms, and interpolation is not available. In addition, most of our results can be extended to degenerate problems. (ii) In [16, Dirichlet type problems are considered, and the authors have to assume extra decay properties of the jump vector $\eta$ near the boundary, conditions that are not satisfied in our Neumann models. 


\section{Assumptions And Definition of SOlutions}

In this section we state the assumptions of problem (1.1) that we will use in the rest of the paper, give the definition of solutions for (1.1), and show that the quantities in this definition are well defined under our assumptions.

We always let $D u(x)$ and $D^{2} u(x)$ denote the gradient and hessian matrix of a function $u$ at $x$, usc and lsc denote upper and lower semicontinuous (functions), $C_{b}$ denotes the space of bounded continuous functions, and $B(x, r), \bar{B}(x, r)$ denotes respectively the open ball $\left\{y \in \mathbb{R}^{N}:|x-y|<r\right\}$ for $x \in \mathbb{R}^{N}$ and $r>0$, and its closure. We also define $P(x, z)=x+\eta(x, z)$. Our assumptions are as follows:

$\left(\mathrm{H}_{f}\right) \quad f \in C_{b}(\bar{\Omega})$.

$\left(\mathrm{H}_{\mu}\right)$ The measure $\mu$ is the sum of two nonnegative Radon measures $\mu_{*}$ and $\mu_{\#}$ on $\mathbb{R}^{N} \backslash\{0\}$,

$$
\mu=c \mu_{*}+\mu_{\#},
$$

where $c$ is either 0 or 1 ,

$$
\int_{|z|<1}\left|z_{N}\right| d \mu_{*}=\infty, \quad \int_{\mathbb{R}^{N}}(1 \wedge|z|) d \mu_{\#}<\infty,
$$

and $\mu_{*}$ satisfies (1.2) and is symmetric in the sense that

$$
\int_{\mathbb{R}^{N}} \chi\left(\sigma_{i} z\right) d \mu_{*}(z)=\int_{\mathbb{R}^{N}} \chi(z) d \mu_{*}(z) \quad \text { for } \quad i=1, \ldots, N,
$$

for all $\mu_{*}$-integrable functions $\chi$ where $\sigma_{i} z=\left(z_{1}, \ldots,-z_{i}, \ldots, z_{N}\right)$.

$\left(\mathrm{H}_{\eta}^{0}\right)$ Neumann problem: $P(x, z)_{N}=x_{N}+\eta(x, z)_{N} \geq 0$ for all $x \in \bar{\Omega}, z \in \mathbb{R}^{N}$, and

$$
\eta(x, z)=z \quad \text { if } \quad x_{N}+z_{N} \geq 0 .
$$

$\left(\mathrm{H}_{\eta}^{1}\right)$ At most linear growth of the jumps: There exists $c_{\eta}>0$ such that

$$
|\eta(x, z)| \leq c_{\eta}|z| \quad \text { for all } \quad x \in \bar{\Omega}, z \in \mathbb{R}^{N} .
$$

$\left(\mathrm{H}_{\eta}^{2}\right)$ Antisymmetry with respect to the $z^{\prime}$-variables: For all $x \in \bar{\Omega}$,

$$
\eta\left(x, \sigma_{i} z\right)=\sigma_{i} \eta(x, z) \quad \text { for } \quad i=1, \ldots, N-1,
$$

where $\sigma_{i}$ is defined in $\left(\mathrm{H}_{\mu}\right)$.

$\left(\mathrm{H}_{\eta}^{3}\right)$ Weak continuity condition: If $x_{n} \rightarrow x$ in $\bar{\Omega}$, then

$$
\eta\left(x_{n}, z\right) \rightarrow \eta(x, z) \text { for } \mu \text {-a.e. } z \text {. }
$$

$\left(\mathrm{H}_{\eta}^{4}\right)$ Continuity in the $x^{\prime}$-variable: There exists $L_{\eta}>0$ such that for all $x^{\prime}, y^{\prime} \in$ $\mathbb{R}^{N-1}, z \in \mathbb{R}^{N}$, and $s>0$,

$$
\left|\eta\left(x^{\prime}, s, z\right)^{\prime}-\eta\left(y^{\prime}, s, z\right)^{\prime}\right| \leq L_{\eta}|z|\left|x^{\prime}-y^{\prime}\right| .
$$

$\left(\mathrm{H}_{\eta}^{5}\right)$ Noncensored cases: Contraction in the $N$-th direction,

$$
\left|P(x, z)_{N}-P(y, z)_{N}\right| \leq\left|x_{N}-y_{N}\right| \quad \text { for all } \quad x, y \in \Omega, z \in \mathbb{R}^{N} .
$$

$\left(\mathrm{H}_{\eta}^{6}\right)$ Censored case: For all $z \neq 0$ and $x \in \Omega$,

$$
\eta(x, z)= \begin{cases}z & \text { if } x_{N}+z_{N} \geq 0 \\ 0 & \text { otherwise }\end{cases}
$$


Remark 2.1. Assumption $\left(\mathrm{H}_{\mu}\right)$ means that we can decompose $\mu$ into a sum of a singular symmetric (in the sense of $\left(\mathrm{H}_{\mu}\right)$ ) Lévy measure and a less singular Lévy measure. This assumption covers the stable, the tempered stable, and the larger class of self-decomposable processes in $\mathbb{R}^{N}$; cf. chapter 1.2 in [1]. In all these cases the Lévy measures satisfy

$$
\frac{d \mu}{d z}=\frac{g(z)}{|z|^{N+\alpha}} \quad \text { for } \quad \alpha \in(0,2)
$$

and $\left(\mathrm{H}_{\mu}\right)$ holds with $c=0$ if $\alpha \in(0,1)$, while if $\alpha \in[1,2)$ and $g$ is Lipschitz in $\bar{B}(0,1)$ and bounded, then $\left(\mathrm{H}_{\mu}\right)$ holds with $c=1$. In the last case we may take

$$
\frac{d \mu_{*}}{d z}=\frac{h(z)}{|z|^{N+\alpha}} \quad \text { and } \quad \frac{d \mu_{\#}}{d z}=\frac{g(z)-h(z)}{|z|^{N+\alpha}} \text { for } h(z):=\min _{|y|=|z|} g(y),
$$

and note that $h$ is symmetric and $g-h$ is nonnegative. More generally, we can consider measures where $\frac{d \mu}{d z}=g(z) \frac{d \mu_{*}}{d z}$ and $\mu_{*}$ is symmetric in the sense of $\left(\mathrm{H}_{\mu}\right)$.

Remark 2.2. The cases (a), (b), (c), and (d) mentioned in the introduction satisfy assumptions $\left(\mathrm{H}_{\eta}^{i}\right)$ for $i=0,1,2,3,4$, where in fact $\left(\mathrm{H}_{\eta}^{4}\right)$ holds with $C=0$. Assumption $\left(\mathrm{H}_{\eta}^{5}\right)$ holds except in case (a), and case (a) is equivalent to $\left(\mathrm{H}_{\eta}^{6}\right)$. Note that $\eta$ is continuous in $x$ for $z \neq 0$ in (b), (c), and (d), while in (a), $\eta$ is continuous except on the codimension 1 hypersurface $\left\{z_{N}=x_{N}\right\}$.

Now we will define generalized solutions in the viscosity sense, and to do that we need the following notation:

$$
I[\phi]=I_{\delta}[\phi]+I^{\delta}[\phi],
$$

where

$$
I^{\delta}[\phi]=\int_{|z| \geq \delta} \phi(x+\eta(x, z))-\phi(x) d \mu(z) .
$$

The $I^{\delta}$-term is well defined for any bounded function $\phi$. For the $I_{\delta}$-term there are two cases, depending on whether $c=0$ or 1 in $\left(\mathrm{H}_{\mu}\right)$. If $c=0$, a Taylor expansion shows that $I_{\delta}[\phi](x)$ is well defined for $\phi \in C^{1}$ and $x \in \Omega$. If $c=1$, and the measure $\mu$ is very singular, we add and subtract a compensator term P.V. $\int_{|z|<\delta} D \phi \cdot \eta d \mu$ and write

$$
I_{\delta}[\phi](x)=\tilde{I}_{\delta}[\phi](x)+\mathrm{P} . \mathrm{V} \cdot \int_{|z|<\delta} D \phi(x) \cdot \eta(x, z) d \mu(z)
$$

for

$$
\tilde{I}_{\delta}[\phi](x):=\int_{|z|<\delta} \phi(x+\eta(x, z))-\phi(x)-D \phi(x) \cdot \eta(x, z) d \mu(z) .
$$

Under our assumptions and $C^{2}$-regularity of $\phi$, a nontrivial argument (see Lemma 2.7 below) shows that these two terms are well defined.

Definition 2.3. Assume that $\left(\mathrm{H}_{\mu}\right),\left(\mathrm{H}_{\eta}^{i}\right)$ for $i=0,1,2$ hold.

(i) A bounded usc function $u$ is a viscosity subsolution to (1.1) if, for any test function $\phi \in C^{2}\left(\mathbb{R}^{N}\right)$ and maximum point $x$ of $u-\phi$ in $\bar{B}\left(x, c_{\eta} \delta\right) \cap \bar{\Omega}$,

$$
\begin{array}{crr} 
& F\left(x, u(x), I_{\delta}[\phi]+I^{\delta}[u]\right) \leq 0 & \text { if } x \in \Omega \quad \text { and } \\
\text { either } & F\left(x, u(x), I_{\delta}[\phi]+I^{\delta}[u]\right) \leq 0 & \text { if } x \in \partial \Omega \text { and } c=0, \\
\text { or } & -\frac{\partial \phi}{\partial x_{N}}(x) \leq 0 & \text { if } x \in \partial \Omega \text { and } c=1 .
\end{array}
$$


(ii) A bounded lsc function $v$ is a viscosity supersolution to (1.1) if, for any test function $\phi \in C^{2}\left(\mathbb{R}^{N}\right)$ and minimum point $x$ of $v-\phi$ in $\bar{B}\left(x, c_{\eta} \delta\right) \cap \bar{\Omega}$,

$$
\begin{array}{clr} 
& F\left(x, v(x), I_{\delta}[\phi]+I^{\delta}[v]\right) \geq 0 & \text { if } x \in \Omega \quad \text { and } \\
\text { either } & F\left(x, v(x), I_{\delta}[\phi]+I^{\delta}[v]\right) \geq 0 & \text { if } x \in \partial \Omega \text { and } c=0, \\
\text { or } & -\frac{\partial \phi}{\partial x_{N}}(x) \geq 0 & \text { if } x \in \partial \Omega \text { and } c=1 .
\end{array}
$$

(iii) A viscosity solution is both a sub- and a supersolution.

Example 2.4. As suggested by one of the referees, in the mirror reflection case (model (d)) problem (1.1) is equivalent to a problem posed in $\mathbb{R}^{N}$ with symmetric data $f$. To make this precise, we take $f \in C^{0, \beta}(\bar{\Omega})$, the space of bounded $\beta$-Hölder continuous functions on $\bar{\Omega}$, and $\mu$ to be the Levy measure associated to the $\frac{\alpha}{2}$ Laplacian, i.e. $d \mu(z)=\frac{c_{\alpha, N} d z}{|z|^{N+\alpha}}$. Here $\beta \in(0,1), \alpha \in(0,2)$, and $c_{\alpha, N}$ is a constant. Then we extend $f$ to $\mathbb{R}^{N}$ by setting

$$
f\left(x^{\prime},-x_{N}\right)=f\left(x^{\prime}, x_{N}\right) \quad \text { for all } \quad x^{\prime} \in \mathbb{R}^{N-1}, x_{N}>0,
$$

and consider the problem

$$
u+(-\Delta)^{\frac{\alpha}{2}} u=f \quad \text { in } \quad \mathbb{R}^{N} .
$$

This problem has a unique viscosity solution (cf. e.g. [6]) satisfying

$$
u\left(x^{\prime},-x_{N}\right)=u\left(x^{\prime}, x_{N}\right) \quad \text { for all } \quad x^{\prime} \in \mathbb{R}^{N-1}, x_{N} \in \mathbb{R} .
$$

By classical Schauder estimates (cf. section V.4.4 in [28]) this solution belongs to $C^{[\alpha+\beta], \alpha+\beta-[\alpha+\beta]}\left(\mathbb{R}^{N}\right)$ and hence is a classical point-wise solution of the equation (cf. [26]). A direct computation using the symmetry of $u$ and continuous differentiability when $\alpha>1$ then shows that $u$ satisfies

$$
\begin{aligned}
& u-I[u]=f \quad \text { in } \quad \Omega \quad \text { for all } \quad \alpha \in(0,2), \beta \in(0,1) \text {, } \\
& \frac{\partial u}{\partial x_{N}}=0 \quad \text { at } \quad \partial \Omega \quad \text { for all } \quad \alpha \in(1,2), \beta \in(0,1) \text {, }
\end{aligned}
$$

where $I$ is defined below (1.1) and $\eta$ is given by model (d).

For $\alpha \in(0,1)$ the solution may not be differentiable at $\partial \Omega$, and hence the Neumann condition will not hold in the classical sense. To see this, let $u(x)=$ $\left|x_{N}\right|^{\alpha+\beta} \phi(x)$, where $\phi \in C_{c}^{\infty}\left(\mathbb{R}^{N}\right)$ and $\phi=1$ in $B(0,1)$. For $\alpha+\beta \in(0,1)$, $u \in C^{0, \alpha+\beta}\left(\mathbb{R}^{N}\right)$, and it follows (cf. [26]) that

$$
f:=u+(-\Delta)^{\frac{\alpha}{2}} u \in C^{0, \beta}\left(\mathbb{R}^{N}\right) .
$$

The normal derivative of $u$ blows up at the boundary, but $u$ satisfies the Neumann condition in the sense of the above definition and in the sense of (1.5).

Remark 2.5. The constant $c_{\eta}$ is defined in $\left(\mathrm{H}_{\eta}^{1}\right)$. If $u$ and $\phi$ are smooth and $x$ is a maximum point of $u-\phi$ over $\bar{B}\left(x, c_{\eta} \delta\right) \cap \bar{\Omega}$, then by $\left(\mathrm{H}_{\eta}^{1}\right)$,

$$
u(x)-\phi(x) \geq u(x+\eta(x, z))-\phi(x+\eta(x, z)) \text { for all }|z|<\delta .
$$

If we rewrite this inequality and integrate, we find formally that $I_{\delta}[u](x) \leq I_{\delta}[\phi](x)$. Lemma 2.7 below makes this computation rigorous. From this inequality it is easy to prove that classical (sub)solutions of (1.1) are viscosity (sub)solutions. Moreover, smooth viscosity (sub)solutions are classical (sub)solutions (simply take $\phi=u$ ). 
Remark 2.6. In general, see e.g. [4,13, boundary conditions in the viscosity sense should be posed for a subsolution $u$ by requiring that for every maximum point $x \in \partial \Omega$ of $u-\phi$ in $\bar{B}\left(x, c_{\eta} \delta\right) \cap \bar{\Omega}$,

$$
\min \left(F\left(x, u, I_{\delta}[\phi]+I^{\delta}[u]\right),-\frac{\partial \phi}{\partial x_{N}}(x)\right) \leq 0 .
$$

This means that either an inequality involving the $F$-equation or the boundary condition holds, and this is also the case for supersolutions where a similar definition applies. In the cases we consider in this paper, such a general definition simplifies to Definition 2.3. If the measure $\mu$ is very singular (case $c=1$ ), then the inequalities involving the $F$-equation cannot hold on the boundary and hence the inequalities have to hold for the boundary condition instead. In the $c=0$ case, on the contrary, the equation will hold up to the boundary and the boundary condition cannot be imposed in general. As already mentioned in the introduction, this is linked to the fact that in the case of reflecting $\alpha$-stable process with $\alpha<1$, the process will never reach the boundary [9]. We also refer to [4] for results in this direction. In other words, we only end up with a Neumann boundary condition if $c=1$, i.e. the measure has a "strong" singular part $\mu_{*}$. In this case the intensity of small jumps is so strong that our jump-reflection mechanisms (e.g. (a) - (d)) are not sufficient to prevent the process from "diffusing" onto the boundary, and we need to add a reflection process at the boundary to keep the process inside (just as in the case of Brownian motion). We also note that the symmetry of $\mu_{*}$ is a natural condition in order to obtain Neumann and not oblique derivative boundary conditions; cf. Lemma 3.3 and its proof.

We now prove that $I_{\delta}[\phi]$ is well defined for $\phi \in C^{2}$.

Lemma 2.7. Assume that $\left(\mathrm{H}_{\mu}\right)$ and $\left(\mathrm{H}_{\eta}^{i}\right)$ for $i=0,1,2$ hold, and let $x \in \Omega$, $\phi \in C^{2}$, and $\delta>0$.

$$
\begin{aligned}
& \text { (a) P.V. } \int_{|z|<\delta} D \phi(x) \cdot \eta(x, z) d \mu(z) \\
& =\int_{|z|<\delta} D \phi(x) \cdot \eta(x, z) d \mu_{\#}(z)+c \int_{x_{N}<|z|<\delta} D \phi(x) \cdot \eta(x, z) d \mu_{*}(z) .
\end{aligned}
$$

(b) $I_{\delta}[\phi](x)$ is well defined and there is $R=R(x, \eta)>0$ such that

$$
I_{\delta}[\phi](x)=o_{\delta}(1)\|\phi\|_{C^{2}(\bar{B}(x, R))} \quad \text { as } \quad \delta \rightarrow 0 .
$$

In the following, we often drop the P.V. notation for such integrals since they may be expressed in terms of converging integrals. Note that the integral over $\left\{x_{N}<|z|<\delta\right\}$ need not vanish since this region exceeds the boundary, and hence $\eta(x, z)$ will not be odd there.

To prove Lemma 2.7 and for later use, we give the following result.

Lemma 2.8. Assume that $\left(\mathrm{H}_{\mu}\right)$ and $\left(\mathrm{H}_{\eta}^{i}\right)$ for $i=0,1,2$ hold.

(i) Let $x \in \Omega, 0<r<\rho \leq x_{N}$, and $v \in \mathbb{R}^{N}$ be a fixed vector. Then

$$
\int_{r<|z|<\rho} v \cdot \eta(x, z) d \mu(z)=\int_{r<|z|<\rho} v \cdot \eta(x, z) d \mu_{\#}(z)
$$

and

$$
\mathrm{P} . \mathrm{V} . \int_{|z|<\rho} v \cdot \eta(x, z) d \mu(z)=\int_{|z|<\rho} v \cdot \eta(x, z) d \mu_{\#}(z) .
$$


(ii) Let $x \in \bar{\Omega}, 0<r<\delta$, and $v^{\prime} \in \mathbb{R}^{N-1}$ be a fixed vector. Then

$$
\int_{r<|z|<\delta} v^{\prime} \cdot \eta(x, z)^{\prime} d \mu(z)=\int_{r<|z|<\delta} v^{\prime} \cdot \eta(x, z)^{\prime} d \mu_{\#}(z)
$$

and

$$
\text { P.V. } \int_{|z|<\delta} v^{\prime} \cdot \eta(x, z)^{\prime} d \mu(z)=\int_{|z|<\delta} v^{\prime} \cdot \eta(x, z)^{\prime} d \mu_{\#}(z) .
$$

(iii) Let $x \in \bar{\Omega}$ and $0<r<\delta$. Then

$$
\int_{r<|z|<\delta} \eta(x, z)_{N} d \mu_{*}(z) \geq \int_{\substack{r<|z|<\delta \\ z_{N}>x_{N}}}\left(z_{N}-x_{N}\right) d \mu_{*}(z) \geq 0 .
$$

Proof. (i) By $\left(\mathrm{H}_{\mu}\right), \mu=c \mu_{*}+\mu_{\#}$ with symmetric (cf. $\left.\left(\mathrm{H}_{\mu}\right)\right) \mu_{*}$. If $|z|<\rho \leq x_{N}$, then $\eta(x, z)=z$ by $\left(\mathrm{H}_{\eta}^{0}\right)$. Hence $\eta$ is odd with respect to the $z$ variable, and the integral with respect to the symmetric part $c \mu_{*}$ is zero. Passing to the limit as $r \rightarrow 0$ and using the integrability of $\mu_{\#}$ in $\left(\mathrm{H}_{\mu}\right)$ along with $\left(\mathrm{H}_{\eta}^{1}\right)$ finishes the proof of (i).

(ii) Let $\sigma^{\prime}=\sigma_{1} \circ \sigma_{2} \circ \cdots \circ \sigma_{N-1}$, i.e. $\sigma^{\prime} z=\left(-z^{\prime}, z_{N}\right)$. Because of the symmetry properties of $\mu_{*}$ and $\eta$ (see $(\mathrm{H})_{\mu}$ and $(\mathrm{H})_{\eta}^{2}$ ),

$$
\int_{r<|z|<\delta} v^{\prime} \cdot \eta\left(x, \sigma^{\prime} z\right)^{\prime} d \mu_{*}(z)=\int_{r<|z|<\delta} v^{\prime} \cdot \eta(x, z)^{\prime} d \mu_{*}(z) .
$$

On the other hand, since $\eta\left(x, \sigma^{\prime} z\right)=\sigma^{\prime} \eta(x, z)$ by $\left(\mathrm{H}_{\eta}^{2}\right)$, the above integral is zero. The result on the principal value is obtained as in the first case, after letting $r \rightarrow 0$.

(iii) Let us decompose

$$
\begin{aligned}
& \int_{r<|z|<\delta} \eta(x, z)_{N} d \mu_{*}(z)=\int_{\substack{r<|z|<\delta \\
-x_{N} \leq z_{N} \leq x_{N}}} \eta(x, z)_{N} d \mu_{*}(z) \\
& \quad+\int_{\substack{r<|z|<\delta \\
z_{N}>x_{N}}} \eta(x, z)_{N} d \mu_{*}(z)+\int_{\substack{r<|z|<\delta \\
z_{N}<-x_{N}}} \eta(x, z)_{N} d \mu_{*}(z) .
\end{aligned}
$$

The integral over $-x_{N} \leq z_{N} \leq x_{N}$ vanishes since $\eta(y, z)=z$ in this region and $\mu_{*}$ is symmetric by $(\mathrm{H})_{\eta}^{2}$. By $\left(\mathrm{H}_{\eta}^{0}\right)$ we have that $\eta(x, z)_{N} \geq-x_{N}$ if $z_{N}<-x_{N}$ and $\eta(x, z)=z_{N}>x_{N}$ if $z_{N}>x_{N}$. Hence by the symmetry of $\mu_{*}$,

$$
\int_{r<|z|<\delta} \eta(x, z)_{N} d \mu_{*}(z) \geq \int_{\substack{r<|z|<\delta \\ z_{N}>x_{N}}}\left(z_{N}-x_{N}\right) d \mu_{*}(z) \geq 0,
$$

and the proof is complete.

Proof of Lemma 2.7. (a) By $\left(\mathrm{H}_{\mu}\right), \mu=c \mu_{*}+\mu_{\#}$. Part (a) now follows since the integral with respect to $\mu_{\#}$ exists by $\left(\mathrm{H}_{\eta}^{1}\right)$, while the integral with respect to $\mu_{*}$ (of course, understood in the sense of P.V.) vanishes over $\bar{B}\left(0, x_{N}\right)$ by Lemma 2.8(i).

(b) We consider $I_{\delta}$ in the form (2.1). Here the compensator term (the P.V. term) is well defined by (a), and since $|z|$ is integrable near the origin for $\mu_{\#}$, this term is $|D \phi(x)| o_{\delta}(1)$ as $\delta \rightarrow 0$.

The $\tilde{I}_{\delta}$-term in (2.1) is well defined since the integrand is smooth and bounded by the function $\frac{1}{2}|z|^{2} \max _{\bar{B}(x, R)}\left|D^{2} \phi\right|$, for $R=\max _{y \in \bar{B}(0, \delta)}|\eta(x, y)|$, which is a $\mu$ integrable function over $\bar{B}(0, \delta)$. Moreover, $\int_{0<|z|<\delta}|z|^{2} d \mu(z)=o_{\delta}(1)$ as $\delta \rightarrow 0$ since $|z|^{2}$ is $\mu$-integrable near 0 . 


\section{Derivation of the boundary value problem - PIDE approach}

In this section we derive the boundary value problems from approximate problems involving a sequence of bounded positive Radon measures $\mu^{k}=1_{\{|z|>1 / k\}} \mu$ for $k \rightarrow+\infty$. Assume that $\left(\mathrm{H}_{\mu}\right)$ holds and let $\mu_{\#}^{k}=1_{\{|z|>1 / k\}} \mu_{\#}$ and $\mu_{*}^{k}=$ $1_{\{|z|>1 / k\}} \mu_{*}$. It then easily follows that

$$
\begin{array}{ll}
\left(\mathrm{H}_{\mu}^{1}\right) & \lim _{k \rightarrow+\infty} \int|z| \wedge 1 d \mu_{\#}^{k}(z)=\int|z| \wedge 1 d \mu_{\#}(z), \\
\left(\mathrm{H}_{\mu}^{2}\right) & \lim _{k \rightarrow+\infty} \int|z|^{2} \wedge 1 d \mu_{*}^{k}(z)=\int|z|^{2} \wedge 1 d \mu_{*}(z), \\
\left(\mathrm{H}_{\mu}^{3}\right) & \lim _{k \rightarrow+\infty} \int\left|z_{N}\right| \wedge 1 d \mu_{*}^{k}(z)=\infty .
\end{array}
$$

The approximation problem we consider is then given by

$$
u(x)-I_{\mu_{k}}[u](x)=f(x) \text { in } \bar{\Omega},
$$

where $f \in C_{b}(\bar{\Omega})$ and, for $\phi \in C_{b}(\bar{\Omega})$,

$$
I_{\mu_{k}}[\phi](x)=\int_{\mathbb{R}^{N}} \phi(x+\eta(x, z))-\phi(x) d \mu^{k}(z) .
$$

Since the measures $\mu^{k}$ are bounded, weak or generalized solutions are not needed, and we alway understand equation (3.1) in the classical, pointwise sense. Now we show that it is well posed in $C_{b}(\bar{\Omega})$ and that its solutions $u_{k}$ are uniformly bounded in $k$.

Lemma 3.1. Assume that $\left(\mathrm{H}_{f}\right),\left(\mathrm{H}_{\mu}\right),\left(\mathrm{H}_{\eta}^{0}\right)$, and $\left(\mathrm{H}_{\eta}^{3}\right)$ hold.

(a) For every $k$, there is a unique pointwise solution $u_{k}$ of (3.1) in $C_{b}(\bar{\Omega})$.

(b) If $u_{k}$ and $v_{k}$ are $C_{b}(\bar{\Omega})$ pointwise sub-and supersolutions of (3.1), then

$$
u_{k} \leq v_{k} \quad \text { in } \quad \bar{\Omega} .
$$

(c) If $u_{k}$ is a pointwise solution of (3.1), then $\left\|u_{k}\right\|_{L^{\infty}(\Omega)} \leq\|f\|_{L^{\infty}(\Omega)}$.

Proof. (a) Let $T: C_{b}(\bar{\Omega}) \rightarrow C_{b}(\bar{\Omega})$ be the operator defined by

$$
T u:=u-\varepsilon\left(u-I_{\mu_{k}}[u]-f\right),
$$

where $0<\varepsilon<\left(1+2\left\|\mu^{k}\right\|_{1}\right)^{-1}$ and $\left\|\mu^{k}\right\|_{1}$ is the total (finite!) mass of the measure $\mu^{k}$. Then $T$ is a contraction in the Banach space $C_{b}(\bar{\Omega})$ since

$$
\begin{aligned}
\|T u-T v\|_{\infty} & \leq(1-\varepsilon)\|u-v\|_{\infty}+2 \varepsilon\left\|\mu^{k}\right\|_{1}\|u-v\|_{\infty} \\
& \leq\left(1-\varepsilon\left(1+2\left\|\mu^{k}\right\|_{1}\right)\right)\|u-v\|_{\infty} \\
& \leq C(k)\|u-v\|_{\infty},
\end{aligned}
$$

and $C(k)<1$. Hence there exists a unique $u_{k} \in C_{b}(\bar{\Omega})$ such that $T u_{k}=u_{k}$, which is equivalent to (3.1).

(b) By the definition of the supremum, we can find a sequence $x_{n} \in \bar{\Omega}$ such that

$$
\sup _{\bar{\Omega}}(u-v)<(u-v)\left(x_{n}\right)+\frac{1}{n} \text {. }
$$


Then by the sub- and supersolution (pointwise) inequalities,

$$
\begin{aligned}
& (u-v)\left(x_{n}\right) \leq I_{\mu_{k}}[u-v]\left(x_{n}\right) \\
& =\int_{\mathbb{R}^{N}}(u-v)\left(x_{n}+\eta\left(x_{n}, z\right)\right)-(u-v)\left(x_{n}\right) d \mu^{k}(z)<\frac{1}{n} \mu^{k}\left(\mathbb{R}^{N}\right) .
\end{aligned}
$$

Sending $n \rightarrow 0$ then leads to $\sup _{\bar{\Omega}}(u-v) \leq 0$, and (b) follows.

(c) Follows from (b) since $\pm\|f\|_{L^{\infty}(\Omega)}$ are super- and subsolutions of (3.1).

The limiting problem can be identified through the half-relaxed limit method.

Theorem 3.2. Assume that $\left(\mathrm{H}_{f}\right),\left(\mathrm{H}_{\mu}\right)$, and $\left(\mathrm{H}_{\eta}^{i}\right)$ for $i=0,1,2,3$ hold. Then the half-relaxed limit functions

$$
\bar{u}(x)=\limsup _{k \rightarrow+\infty, y \rightarrow x} u_{k}(y) \quad \text { and } \quad \underline{u}(x)=\liminf _{k \rightarrow+\infty, y \rightarrow x} u_{k}(y)
$$

are respectively sub- and supersolutions of the Neumann boundary problem in the sense of Definition 2.3 .

In the proof we will need the following result whose proof is given at the end of this section.

Lemma 3.3. Assume that $\left(\mathrm{H}_{\eta}^{i}\right)$ for $i=0,1,2$ hold, that $\left(\mathrm{H}_{\mu}\right)$ holds with $c=1$, and let $\delta>0$ and $\gamma_{\mu_{k}, \delta}(y):=\int_{|z|<\delta} \eta(y, z) d \mu_{k}(z)$. If $y_{k} \in \bar{\Omega}$ for $k \in \mathbb{N}$ and $y_{k} \rightarrow x \in \partial \Omega$ as $k \rightarrow \infty$, then

$$
\left|\gamma_{\mu_{k}, \delta}\left(y_{k}\right)\right| \rightarrow \infty \quad \text { and } \quad \frac{\gamma_{\mu_{k}, \delta}\left(y_{k}\right)}{\left|\gamma_{\mu_{k}, \delta}\left(y_{k}\right)\right|} \rightarrow-\mathbf{n},
$$

where $\mathbf{n}=(0,0, \ldots, 0,-1)$ is an outward normal vector of $\partial \Omega$.

Proof of Theorem 3.2. Since the proofs are similar for $\bar{u}$ and $\underline{u}$, we only show the one for $\bar{u}$. Let $\delta>0$ and $\phi \in C^{2}$, and assume that $\bar{u}-\phi$ has a maximum point $x$ in $\bar{B}\left(x, c_{\eta} \delta\right) \cap \bar{\Omega}$. By modifying the test function if necessary, we may always assume that the maximum is strict. A standard argument then shows that for $k$ large enough, $u_{k}-\phi$ has a maximum point $y_{k}$ in $\bar{B}\left(x, c_{\eta} \delta\right) \cap \bar{\Omega}$, and when $k \rightarrow+\infty$,

$$
y_{k} \rightarrow x \quad \text { and } \quad u_{k}\left(y_{k}\right) \rightarrow \bar{u}(x) .
$$

For a proof, we refer e.g. to Lemma 4.2 in [3] or Lemma V.1.6 in [2].

Case 1: $x \in \Omega$, i.e. $x_{N}>0$. Here we may assume that $y_{k} \in \Omega$. Let $\delta_{k}=\delta-\left|x-y_{k}\right|$ and $0<r \leq \delta_{k}$, and note that $\bar{B}\left(y_{k}, c_{\eta} r\right) \subset \bar{B}\left(x, c_{\eta} \delta\right)$. Since the maximum of $\left(u_{k}-\phi\right)$ in $\bar{B}\left(y_{k}, c_{\eta} r\right)$ is attained at $y_{k}$, we find that

$$
\begin{aligned}
& \left(I_{\mu_{k}}\right)_{r}\left[u_{k}\right]\left(y_{k}\right):=\int_{|z|<r} u_{k}\left(y_{k}+\eta\left(y_{k}, z\right)\right)-u_{k}\left(y_{k}\right) d \mu^{k} \\
& \leq \int_{|z|<r} \phi\left(y_{k}+\eta\left(y_{k}, z\right)\right)-\phi\left(y_{k}\right) d \mu^{k}=\left(I_{\mu_{k}}\right)_{r}[\phi]\left(y_{k}\right) .
\end{aligned}
$$

Since $u_{k}$ is a pointwise solution of (3.1), for all $0<r \leq \delta_{k}$,

$$
u_{k}\left(y_{k}\right)-\left(I_{\mu_{k}}\right)_{r}[\phi]\left(y_{k}\right)-\left(I_{\mu_{k}}\right)^{r}\left[u_{k}\right]\left(y_{k}\right) \leq f\left(y_{k}\right),
$$

where $\left(I_{\mu_{k}}\right)^{r}\left[u_{k}\right](x):=\int_{|z| \geq r} u_{k}(x+\eta(x, z))-u_{k}(x) d \mu^{k}(z)$. 
We want to pass to the limit in this inequality and consider first the $\left(I_{\mu_{k}}\right)^{r}$-term. By the definition of $\bar{u}$ and $\left(\mathrm{H}_{\eta}^{3}\right)$,

$$
\limsup _{k \rightarrow+\infty} u_{k}\left(y_{k}+\eta\left(y_{k}, z\right)\right) \leq \bar{u}(x+\eta(x, z)) \text { for a.e. } z \text {. }
$$

Since we integrate away from the singularity of $\mu$, we can use Fatou's Lemma and $\left(\mathrm{H}_{\mu}^{1}\right)$ and $\left(\mathrm{H}_{\mu}^{2}\right)$ to show that

$$
\limsup _{k \rightarrow \infty}\left(I_{\mu_{k}}\right)^{r}\left[u_{k}\right]\left(y_{k}\right) \leq \int_{|z|>r} \bar{u}(x+\eta(x, z))-\bar{u}(x) d \mu(z)=I^{r}[\bar{u}](x) .
$$

To pass to the limit in the $\left(I_{\mu_{k}}\right)_{r}$-term, we write it as

$$
\begin{aligned}
& \left(I_{\mu_{k}}\right)_{r}[\phi]\left(y_{k}\right) \\
& =\underbrace{\int_{|z|<r} \phi\left(x+\eta\left(y_{k}, z\right)\right)-\phi\left(y_{k}\right)-D \phi\left(y_{k}\right) \cdot \eta\left(y_{k}, z\right) d \mu^{k}(z)}_{=\left(\tilde{I}_{\mu_{k}}\right)_{r}[\phi]\left(y_{k}\right)}+\gamma_{\mu_{k}, r}\left(y_{k}\right) \cdot D \phi\left(y_{k}\right),
\end{aligned}
$$

where $\gamma_{\mu_{k}, r}(x):=\int_{|z|<r} \eta(x, z) d \mu^{k}(z)$. For $|z|<r$, a Taylor expansion then yields

$$
\left|\phi\left(y_{k}+\eta\left(y_{k}, z\right)\right)-\phi\left(y_{k}\right)-D \phi\left(y_{k}\right) \cdot \eta\left(y_{k}, z\right)\right| \leq\left\|D^{2} \phi\right\|_{L^{\infty}\left(\bar{B}\left(x, c_{\eta} r\right)\right)}|z|^{2} .
$$

Hence by $\left(\mathrm{H}_{\eta}^{1}\right),\left(\mathrm{H}_{\eta}^{3}\right),\left(\mathrm{H}_{\mu}^{1}\right)$ and $\left(\mathrm{H}_{\mu}^{2}\right)$, we can use the Dominated Convergence Theorem to show that

$$
\left(\tilde{I}_{\mu_{k}}\right)_{r}[\phi]\left(y_{k}\right) \rightarrow \int_{|z|<r} \phi(x+\eta(x, z))-\phi(x)-\eta(x, z) D \phi(x) d \mu(z)=\tilde{I}_{r}[\phi](x) .
$$

Next, by Lemma 2.7

$$
\gamma_{\mu_{k}, r}\left(y_{k}\right)=\int_{|z|<r} \eta\left(y_{k}, z\right) d \mu_{\#}^{k}(z)+c \int_{y_{k, N} \leq|z|<r} \eta\left(y_{k}, z\right) d \mu_{*}^{k}(z),
$$

where the last integral is understood to be zero if $y_{k, N}>r$. Note that since $y_{k, N} \rightarrow x_{N}>0$, the domain of integration of the $\mu_{*}$-integral is always bounded away from $z=0$ when $k$ is large. Along with $\left(\mathrm{H}_{\eta}^{3}\right)$ and $\left(\mathrm{H}_{\mu}^{2}\right)$, this allows us to pass to the limit in the $\mu_{*}$-integral using the Dominated Convergence Theorem. Similarly, we may pass to the limit in the $\mu_{\#}$-integral by $\left(\mathrm{H}_{\eta}^{1}\right),\left(\mathrm{H}_{\eta}^{3}\right)$ and $\left(\mathrm{H}_{\mu}^{1}\right)$. We find that

$$
\begin{aligned}
\gamma_{\mu_{k}, r}\left(y_{k, N}\right) \rightarrow \gamma_{r}(x) & :=\int_{|z|<r} z d \mu_{\#}(x)+c \int_{x_{N} \leq|z|<r} \eta(x, z) d \mu_{*}(x) \\
& =\text { P.V. } \int_{|z|<r} \eta(x, z) d \mu(x)=: \gamma_{r}(x),
\end{aligned}
$$

where we used Lemma 2.7 again. Hence we can conclude that

$$
\lim _{k \rightarrow \infty}\left(I_{\mu_{k}}\right)_{r}[\phi]\left(y_{k}\right)=\tilde{I}_{r}[\phi]\left(y_{k}\right)+\gamma_{r}(x) \cdot D \phi(x)=I_{r}[\phi](x) .
$$

Since $\delta_{k} \rightarrow \delta$, we end up with the following limit equation:

$$
\bar{u}(x)-I_{r}[\phi](x)-I^{r}[\bar{u}](x) \leq f(x)
$$

for every $0<r<\delta$. Using the Dominated Convergence Theorem again, we send $r \rightarrow \delta$ and obtain the subsolution condition for (1.1) at the point $x \in \Omega$. 
Case 2: $x \in \partial \Omega$, i.e. $x_{N}=0$. We first show it in the case $c=1$. Following the argument in Case 1, we may rewrite the subsolution condition as

$$
\frac{u_{k}\left(y_{k}\right)-\left(\tilde{I}_{\mu_{k}}\right)_{\delta}[\phi]\left(y_{k}\right)-\left(I_{\mu_{k}}\right)^{\delta}\left[u_{k}\right]\left(y_{k}\right)-f\left(y_{k}\right)}{\left|\gamma_{\mu_{k}, \delta}\left(y_{k}\right)\right|}-\frac{\gamma_{\mu_{k}, \delta}\left(y_{k}\right) \cdot D \phi\left(y_{k}\right)}{\left|\gamma_{\mu_{k}, \delta}\left(y_{k}\right)\right|} \leq 0 .
$$

Note that this time it may happen that $y_{k} \in \partial \Omega$. By Lemma 3.3. $\left|\gamma_{\mu_{k}, \delta}\left(y_{k}\right)\right| \rightarrow \infty$, and since $u_{k}$ and $f$ are uniformly bounded,

$$
\frac{u_{k}\left(y_{k}\right)}{\left|\gamma_{\mu_{k}, \delta}\left(y_{k}\right)\right|}, \quad \frac{f\left(y_{k}\right)}{\left|\gamma_{\mu_{k}, \delta}\left(y_{k}\right)\right|}, \quad \frac{\left(I_{\mu_{k}}\right)^{\delta}\left[u_{k}\right]\left(y_{k}\right)}{\left|\gamma_{\mu_{k}, \delta}\left(y_{k}\right)\right|}
$$

all converge to zero. The same is true for

$$
\frac{\left(\tilde{I}_{\mu_{k}}\right)_{\delta}[\phi]\left(y_{k}\right)}{\left|\gamma_{\mu_{k}, \delta}\left(y_{k}\right)\right|}
$$

since the integrand of the numerator is controlled by $\left\|D^{2} \phi\right\|_{\infty}|z|^{2} 1_{\{|z|<\delta\}}$ and $\mu^{k}$ satisfy $\left(\mathrm{H}_{\mu}^{1}\right)$ uniformly in $k$. Using Lemma 3.3 again, we have $\gamma_{\mu_{k}, \delta}\left(y_{k}\right) /\left|\gamma_{\mu_{k}, \delta}\left(y_{k}\right)\right| \rightarrow$ $-\mathbf{n}$, so that we may go to the limit in the above inquality to find that

$$
-\frac{\partial \phi}{\partial x_{N}}(x)=\frac{\partial \phi}{\partial \mathbf{n}}(x) \leq 0 .
$$

In the case when $c=0$, the measure $\mu=\mu_{\#}$, which is less singular than $\mu_{*}$. The same line of arguments as in the proof for $x \in \Omega$ (much easier this time) now shows that the equation holds at $x \in \partial \Omega$.

Proof of Lemma 3.3. First note that by Lemma 2.8(ii) with $y_{k}$ instead of $x$ and $\mu^{k}$ instead of $\mu$,

$$
\gamma_{\mu_{k}, \delta}\left(y_{k}\right)^{\prime}=\int_{|z|<\delta} \eta\left(y_{k}, z\right)^{\prime} d \mu^{k}(z)=\int_{|z|<\delta} \eta\left(y_{k}, z\right)^{\prime} d \mu_{\#}^{k}(z),
$$

which remains uniformly bounded in $k$ because of $\left(\mathrm{H}_{\eta}^{1}\right)$ and our assumption on $\mu_{\#}$. Since $y_{k, N} \rightarrow x_{N}=0$, we can assume that $0 \leq y_{k, N}<\delta$, and by $\left(\mathrm{H}_{\mu}\right)$,

$$
\left(\gamma_{\mu_{k}, \delta}\right)_{N}\left(y_{k}\right)=\int_{|z|<\delta} \eta\left(y_{k}, z\right)_{N} d \mu_{\#}^{k}(z)+\int_{|z|<\delta} \eta\left(y_{k}, z\right)_{N} d \mu_{*}^{k}(z) .
$$

As above, the first integral is uniformly bounded as $k \rightarrow \infty$. For the second one, we send $r \rightarrow 0$ in Lemma 2.8(iii) to find that

$$
\int_{|z|<\delta} \eta\left(y_{k}, z\right)_{N} d \mu_{*}^{k}(z) \geq \int_{\substack{|z|<\delta \\ y_{k, N}<z_{N}}}\left(z_{N}-y_{k, N}\right) d \mu_{*}^{k}(z) \geq 0,
$$

and, since $y_{k, N} \rightarrow 0$, we can then use Fatou's Lemma to show that

$$
\int_{\substack{|z|<\delta \\ z_{N}>0}} z_{N} d \mu_{*}(z) \leq \liminf _{k \rightarrow \infty} \int_{\substack{|z|<\delta \\ y_{k, N}<z_{N}}}\left(z_{N}-y_{k, N}\right) d \mu_{*}^{k}(z) .
$$

Applying symmetry of the measure $\mu_{*}$ along with $\left(\mathrm{H}_{\mu}^{3}\right)$, we are led to

$$
\int_{\substack{|z|<\delta \\ z_{N}>0}} z_{N} d \mu_{*}(z)=\frac{1}{2} \int_{|z|<\delta}\left|z_{N}\right| d \mu_{*}(z)=+\infty .
$$


Hence we have proved that $\left(\gamma_{\mu_{k}, \delta}\right)_{N}\left(y_{k}\right) \rightarrow \infty$ as $k \rightarrow \infty$, and if we use that $\left(\gamma_{\mu_{k}, \delta}\right)^{\prime}\left(y_{k}\right)$ is uniformly bounded, we see that

$$
\frac{\gamma_{\mu_{k}, \delta}\left(y_{k}\right)}{\left|\gamma_{\mu_{k}, \delta}\left(y_{k}\right)\right|}=\left(\frac{\left(\gamma_{\mu_{k}, \delta}\right)^{\prime}\left(y_{k}\right)}{\left|\gamma_{\mu_{k}, \delta}\left(y_{k}\right)\right|}, \frac{\left(\gamma_{\mu_{k}, \delta}\right)_{N}\left(y_{k}\right)}{\left|\gamma_{\mu_{k}, \delta}\left(y_{k}\right)\right|}\right) \longrightarrow(0,0, \ldots, 0,1)=-\mathbf{n} .
$$

\section{Comparison in NONCENSORED CASES}

In this section we prove a comparison result for the noncensored cases, i.e. under assumptions $\left(\mathrm{H}_{\eta}^{i}\right)$ for $i=0, \ldots, 5$. These assumptions cover all the examples given in the introduction, except example (a) - the censored case. As a conseqence of the comparison result and the results of the previous sections, we also obtain wellposedness for (1.1). The comparison result is the following

Theorem 4.1. Assume that $\left(\mathrm{H}_{\mu}\right),\left(\mathrm{H}_{f}\right)$, and $\left(\mathrm{H}_{\eta}^{i}\right)$ for $i=0, \ldots, 5$ hold, and let $f, g \in C_{b}(\bar{\Omega})$. Let $u$ be a bounded usc subsolution of (1.1) with data $f$ and $v a$ bounded lsc supersolution of (1.1) with data $g$. If $f \leq g$ in $\bar{\Omega}$, then $u \leq v$ in $\Omega$.

From this result it follows that the half-relaxed limits in Theorem 3.2 satisfy $\bar{u} \leq \underline{u}$ in $\bar{\Omega}$. Since the opposite inequality is always satisfied, this means that $u:=\bar{u}=\underline{u}$ is the solution of (1.1) according to Definition 2.3. Uniqueness and continuous dependence (on $f$ ) follows from Theorem 4.1 by standard arguments (cf. [13]), and we have the following result:

Corollary 4.2. Under the assumptions of Theorem 4.1, there exists a unique viscosity solution of (1.1) depending continuously on $f$.

Proof of Theorem 4.1. We argue by contradiction assuming that $M:=\sup _{\bar{\Omega}}(u-$ $v)>0$. We provide the full details only when $c=1$. The case $c=0$ is much easier since the equation then holds, even on the boundary.

To get a contradiction, we introduce the function

$$
\Psi_{R}(x):=u(x)-v(x)-\psi_{R}(x, x),
$$

where $\psi_{R}$ is given by

$$
\psi_{R}(x, y):=\psi\left(\left|x_{N}\right| / R\right)+\psi\left(\left|y_{N}\right| / R\right)+\psi\left(\left|x^{\prime}\right| / R\right)+\psi\left(\left|y^{\prime}\right| / R\right)
$$

and $\psi$ is a smooth function such that

$$
\psi(s)= \begin{cases}0 & \text { for } 0 \leq s<1 / 2 \\ \text { increasing } & \text { for } 1 / 2 \leq s<1 \\ \|u\|_{\infty}+\|v\|_{\infty}+1 & \text { for } s \geq 1\end{cases}
$$

Let $M_{R}:=\sup \Psi_{R}$ and note that $M_{R} \rightarrow M$ as $R \rightarrow \infty$. Since $\Psi_{R}(x) \leq-2$ for $|x|$ large and $M>0$, the function $\Psi_{R}$ achieves its positive maximum $M_{R}>\frac{M}{2}$ at a point $\bar{x}_{R}$ for $R$ large enough. There are two cases for such large $R>0$ :

(a) either there is a maximum point $\bar{x}_{R}$ of $\Psi_{R}$ not located on the boundary,

(b) or all maximum points $\bar{x}_{R}$ of $\Psi_{R}$ are located on the boundary.

Case (a). The proof is rather classical, so we just sketch it and refer to [6] and to the proof of case (b) for more precise arguments. First, to ensure that $\bar{x}_{R}$ is the unique maximum point, we replace $\Psi_{R}$ by the function $x \mapsto \Psi_{R}(x)-\sigma\left|x-\bar{x}_{R}\right|^{4}$ 
for $0<\sigma \ll 1$. Then to get a comparison result, we do a doubling of variables argument considering the maximum of $u(x)-v(y)-\phi(x, y)$, where the test function

$$
\phi(x, y)=\frac{\left|x^{\prime}-y^{\prime}\right|^{2}}{\varepsilon^{\prime 2}}+\frac{\left|x_{N}-y_{N}\right|^{2}}{\varepsilon_{N}^{2}}+\psi_{R}(x, y)+\sigma\left|x-\bar{x}_{R}\right|^{4}
$$

for $\varepsilon^{\prime}, \varepsilon_{N}>0$. The separate treatment of the $N$-components will allow us to exploit $\left(\mathrm{H}_{\eta}^{5}\right)$, the contraction property of the $N$-components of $\eta$. We note that in all ensuing viscosity inequalities (see Definition 2.3), $\phi$ will only be integrated over compact sets, so the integrability at infinity of $|x|^{2}$ and $|x|^{4}$ with respect to the measure $\mu$ is not an issue. Moreover, the contribution from the $\sigma$-term will be small for small $\sigma$.

In this setup, the maximum points $(\bar{x}, \bar{y})$ of $u-v-\phi$ converge as $\varepsilon^{\prime}, \varepsilon_{N} \rightarrow 0$ to $\left(\bar{x}_{R}, \bar{x}_{R}\right)$, and hence they are bounded away from the boundary for $\varepsilon^{\prime}, \varepsilon_{N}$ small enough. This last property implies that we can use directly the equation (the viscosity inequalites for $F$ in Definition 2.3) and obtain $\max \left(\Psi_{R}-\sigma\left|x-\bar{x}_{R}\right|^{4}\right) \leq o(1)$ as $\sigma \rightarrow 0, R \rightarrow \infty$, which is a contradiction to $\max \Psi_{R}>0$ for $R$ large.

Case (b). We do a doubling of variables argument considering the maximum of

$$
\Phi(x, y):=u(x)-v(y)-\phi(x, y),
$$

with a new test function

$$
\phi(x, y):=\frac{\left|x^{\prime}-y^{\prime}\right|^{2}}{\varepsilon^{\prime 2}}+\frac{\left|x_{N}-y_{N}\right|^{2}}{\varepsilon_{N}^{2}}+\psi_{R}(x, y)-\mathrm{d}_{\nu}\left(x_{N}\right)-\mathrm{d}_{\nu}\left(y_{N}\right),
$$

for $\varepsilon, \varepsilon^{\prime}>0,0<\nu<1, \mathrm{~d}_{\nu}(\cdot)=\nu \mathrm{d}(\cdot)$ for a smooth function $d$ satisfying

$$
\mathrm{d}(s)= \begin{cases}s & \text { for } 0 \leq s<1 / 2, \\ \text { is increasing } & \text { for } 1 / 2 \leq s<1 \\ 1 & \text { for } s \geq 1\end{cases}
$$

The term $\mathrm{d}_{\nu}$ plays the role of a distance to the boundary of the domain. Such terms are common in Neumann proofs to prevent the maximum points from being on the boundary (cf. Step 1 below).

If $0<\nu<1$ and $R \gg 1$ are fixed, then $\Phi \leq 0$ for $|x|,|y|$ large enough, while $\Phi\left(\bar{x}_{R}, \bar{x}_{R}\right)=\sup \Psi_{R}=M_{R}>0$ for any maximum point $\bar{x}_{R}$ of $\Psi_{R}$. Recall that we are in Case (b) where $\mathrm{d}_{\nu}\left(\left(\bar{x}_{R}\right)_{N}\right)=0$. Hence the maximum of $\Phi$ is attained at some point $\bar{\Omega}^{2}$ which we just denote by $(x, y)$ for simplicity. Note that $x, y, M_{R}$ now depend on $\varepsilon^{\prime}, \varepsilon_{N}, \nu, R$. At the end of the proof, we are going to first let $\varepsilon_{N} \rightarrow 0$, then $\varepsilon^{\prime} \rightarrow 0$, then $\nu \rightarrow 0$, and finally $R \rightarrow \infty$.

By rearranging the maximum point inequalities

$$
\Phi(x, x)+\Phi(y, y) \leq 2 \Phi(x, y) \quad \text { and } \quad \Phi\left(x_{R}, x_{R}\right) \leq \Phi(x, y),
$$

and using the boundedness and semicontinuity of $u, v$ and definition of $\psi_{R}$, we find that

$$
\left|x_{N}-y_{N}\right| \leq \bar{c} \varepsilon_{N}, \quad\left|x^{\prime}-y^{\prime}\right| \leq \bar{c} \varepsilon^{\prime}, \quad|x|,|y|<R,
$$

where $\bar{c}:=4\left(\|u\|_{\infty}+\|v\|_{\infty}\right)$, and

$$
2 \frac{\left|x^{\prime}-y^{\prime}\right|^{2}}{\varepsilon^{\prime 2}} \leq u(x)-u(y)-(v(y)-v(x))=o_{\varepsilon_{N}, \varepsilon^{\prime}}(1),
$$


where $o_{\varepsilon_{N}, \varepsilon^{\prime}}(1)$ means that after passing to the limit as $\varepsilon_{N} \rightarrow 0$, we are left with a quantity which is an $o_{\varepsilon^{\prime}}(1)$. From this it follows that as $\varepsilon_{N}, \varepsilon^{\prime}, \nu \rightarrow 0$,

$$
\Phi(x, y) \rightarrow \sup \Psi_{R}=M_{R} \quad \text { and (by taking subsequences) } \quad x, y \rightarrow x_{R},
$$

where $x_{R}$ is a maximum point of $\Psi_{R}$. Since $x_{R}$ is at the boundary by assumption, we assume without loss of generality that

$$
0 \leq x_{N}, y_{N}<1
$$

Finally, we also get that

$$
u(x)-v(y)=M+o_{\varepsilon_{N}, \varepsilon^{\prime}, \nu, R}(1)
$$

as $\varepsilon_{N}, \varepsilon^{\prime}, \nu \rightarrow 0$ first and then $R \rightarrow \infty$ at the end. We now proceed with the proof in several steps.

Step 1. Pushing the maximum points inside. We prove that the $F$-viscosity inequalities hold for $u$ and $v$. According to Definition 2.3. this is clearly the case if $c=0$ since these viscosity inequalities hold even if the maximum or minimum points are on the boundary.

Assume that $c=1$ and e.g. $x_{N}=0$. Then $x$ is a (global) maximum point of the function $z \mapsto u(z)-v(y)-\varphi(z, y)$, so $-\frac{\partial \varphi}{\partial x_{N}}(x, y) \leq 0$ by Definition 2.3. But since $\frac{\partial \psi_{R}}{\partial x_{N}}$ is zero in a neighborhood of the boundary by definition,

$$
-\frac{\partial \varphi}{\partial x_{N}}(x, y)=-\frac{2\left(x_{N}-y_{N}\right)}{\varepsilon_{N}^{2}}-\frac{\partial \psi_{R}}{\partial x_{N}}(x, y)+\frac{d}{d s} \mathrm{~d}_{\nu}(0)=\frac{2 y_{N}}{\varepsilon_{N}^{2}}+\nu>0
$$

which is a contradiction. Therefore $x_{N}$ cannot be zero. A similar argument shows that $y_{N}>0$ as well, and hence both $x$ and $y$ are inside $\Omega$.

Step 2. Writing the viscosity inequalities and sending $\delta$ to zero. Let

$$
\delta<\rho:=\min \left(x_{N}, y_{N}\right)
$$

Since $u$ is a viscosity subsolution and the function $u(\cdot)-v(y)-\varphi(\cdot, y)$ has a maximum at $x$, by Definition 2.3(i) and Lemma 2.7.

$$
\begin{aligned}
& u(x)-\int_{|z|<\delta}\left[\varphi(P(x, z), y)-\varphi(x, y)-D_{x} \varphi(x, y) \cdot \eta(x, z)\right] d \mu(z) \\
& - \text { P.V. } \int_{|z|<\delta} D_{x} \varphi(x, y) \eta(x, z) d \mu(z)-\int_{|z| \geq \delta}[u(P(x, z))-u(x)] d \mu(z) \leq f(x) .
\end{aligned}
$$

By Definition 2.3(ii), a similar inequality holds for the supersolution $v$ and the minimum point $y$ of $v(\cdot)-u(x)+\varphi(x, \cdot)$. If we subtract these two inequalities and 
use Lemma 2.8(i), then

$$
\begin{aligned}
& -\int_{|z|<\delta}\left[\varphi(P(x, z), y)-\varphi(x, y)-D_{x} \varphi(x, y) \cdot \eta(x, z)\right] d \mu(z) \\
& -D_{x} \varphi(x, y) \cdot \int_{|z|<\delta} \eta(x, z) d \mu_{\#}(z) \\
& -\int_{|z|<\delta}\left[\varphi(x, P(y, z))-\varphi(x, y)+D_{y} \varphi(x, y) \cdot \eta(y, z)\right] d \mu(z) \\
& +D_{y} \varphi(x, y) \cdot \int_{|z|<\delta} \eta(y, z) d \mu_{\#}(z) \\
& -\int_{|z| \geq \delta}[u(P(x, z))-v(P(y, z))-u(x)+v(y)] d \mu(z) \\
& +u(x)-v(y) \leq f(x)-f(y) .
\end{aligned}
$$

In order to pass to the limit as $\delta \rightarrow 0$ to get rid of the test function $\varphi$, we use Lemma 2.7 for all the terms which are smooth functions: the integrals over $\bar{B}(0, \delta)$ all vanish as $\delta \rightarrow 0$ and we are left with limit of the integral over $\{|z|>\delta\}$. To this end, we split this integral into two integrals, one over $\{|z| \geq 1\}$ (which is independent of $\delta$ of course) and the other over $\{\delta \leq|z|<1\}$, that we have to deal with.

Using the definition of the maximum point for $\Phi$, we have that for any $z$

$$
u(P(x, z))-v(P(y, z))-\varphi(P(x, z), P(y, z)) \leq u(x)-v(y)-\varphi(x, y) .
$$

Hence, it follows that

$$
\begin{aligned}
& u(P(x, z))-v(P(y, z))-(u(x)-v(y)) \\
& \leq \frac{\left|P(x, z)_{N}-P(y, z)_{N}\right|^{2}}{\varepsilon_{N}^{2}}-\frac{\left|x_{N}-y_{N}\right|^{2}}{\varepsilon_{N}^{2}}+\frac{\left|P(x, z)^{\prime}-P(y, z)^{\prime}\right|^{2}}{\varepsilon^{\prime 2}}-\frac{\left|x^{\prime}-y^{\prime}\right|^{2}}{\varepsilon^{\prime 2}} \\
& \quad+\psi_{R}(P(x, z), P(y, z))-\psi_{R}(x, y) \\
& \quad-\mathrm{d}_{\nu}\left(P(x, z)_{N}\right)+\mathrm{d}_{\nu}\left(x_{N}\right)-\mathrm{d}_{\nu}\left(P(y, z)_{N}\right)+\mathrm{d}_{\nu}\left(y_{N}\right),
\end{aligned}
$$

and we put this inequality into the integral over $\{\delta \leq|z|<1\}$ which gives rise to several terms denoted by (with obvious notation)

$$
\int_{\delta \leq|z|<1}\{u(P(x, z))-v(P(y, z))-u(x)+v(y)\} d \mu(z) \leq T_{\varepsilon_{N}}^{\delta}+T_{\varepsilon^{\prime}}^{\delta}+T_{\psi_{R}}^{\delta}+T_{\mathrm{d}_{\nu}}^{\delta} .
$$

By $\left(\mathrm{H}_{\eta}^{5}\right)$, it follows that $T_{\varepsilon_{N}}^{\delta} \leq 0$. Next,

$$
\begin{aligned}
T_{\varepsilon^{\prime}}^{\delta}= & \int_{\delta \leq|z|<1}\left(\frac{\left|P(x, z)^{\prime}-P(y, z)^{\prime}\right|^{2}}{\varepsilon^{\prime 2}}-\frac{\left|x^{\prime}-y^{\prime}\right|^{2}}{\varepsilon^{\prime 2}}\right) d \mu \\
= & \frac{1}{\varepsilon^{\prime 2}} \int_{\delta \leq|z|<1}\left|\eta(x, z)^{\prime}-\eta(y, z)^{\prime}\right|^{2} d \mu(z) \\
& +\frac{2}{\varepsilon^{\prime 2}} \int_{\delta \leq|z|<1}\left(x^{\prime}-y^{\prime}\right) \cdot\left(\eta(x, z)^{\prime}-\eta(y, z)^{\prime}\right) d \mu(z) .
\end{aligned}
$$

For the first term of $T_{\varepsilon^{\prime}}^{\delta}$, we use the domination of the integrand by $c|z|^{2}$ to pass to the limit as $\delta \rightarrow 0$. For the second one, we use Lemma 2.8(iii) which allows us to 
wipe out the symmetric $\mu_{*}$-contribution, so that we get in the limit

$$
\begin{aligned}
\limsup _{\delta \rightarrow 0} T_{\varepsilon^{\prime}}^{\delta} \leq & \frac{1}{\varepsilon^{\prime 2}} \int_{0<|z|<1}\left|\eta(x, z)^{\prime}-\eta(y, z)^{\prime}\right|^{2} d \mu(z) \\
& +\frac{2}{\varepsilon^{\prime 2}} \int_{0<|z|<1}\left(x^{\prime}-y^{\prime}\right) \cdot\left(\eta(x, z)^{\prime}-\eta(y, z)^{\prime}\right) d \mu_{\#}(z) .
\end{aligned}
$$

We now concentrate on the penalisation terms which are given by integrals of smooth functions. Recall that $0<x_{N}, y_{N}<1$, so that $\psi\left(x_{N} / R\right)$ and $\psi\left(y_{N} / R\right)$ vanish (we have assumed that $R \gg 1$ ). Hence, using Lemma 2.7 we get as $\delta \rightarrow 0$ the following two contributions:

$$
\begin{aligned}
& \lim _{\delta \rightarrow 0} T_{\mathrm{d}_{\nu}}^{\delta}=-\tilde{I}_{1}\left[\mathrm{~d}_{\nu}\right](x)-\frac{d}{d s} \mathrm{~d}_{\nu}\left(x_{N}\right) \mathrm{P} . \mathrm{V} . \int_{0<|z|<1} \eta(x, z)_{N} d \mu(z)+(\cdots)(y), \\
& \lim _{\delta \rightarrow 0} T_{\psi_{R}}^{\delta} \leq-\tilde{I}_{1}\left[\tilde{\psi}_{R}\right](x)-D \tilde{\psi}_{R}(x)^{\prime} \cdot \mathrm{P} . \mathrm{V} . \int_{0<|z|<1} \eta(x, z)_{N} d \mu(z)+(\cdots)(y) .
\end{aligned}
$$

where $\tilde{\psi}_{R}(x):=\psi\left(\left|x^{\prime}\right| / R\right)$ and the $(\cdots)(y)$ notation stands for the same terms but calculated at $y$ instead of $x$. Now, note that $\frac{d}{d s} \mathrm{~d}\left(x_{N}\right)>0$ and use Lemma 2.8 (iii) (with $r=x_{N}>0$ ). This gives that in the principal value for $T_{\mathrm{d}_{\nu}}$, the $\mu_{*}$-term which is multiplied by $(-\nu)$ has a nonpositive contribution. Hence we find that

$$
\lim _{\delta \rightarrow 0} T_{\mathrm{d}_{\nu}}^{\delta} \leq-\nu \tilde{I}_{1}[\mathrm{~d}](x)-\nu \frac{d}{d s} \mathrm{~d}\left(x_{N}\right) \int_{0<|z|<1} \eta(x, z)_{N} d \mu_{\#}(z)+(\cdots)(y)=o_{\nu}(1) .
$$

As for the $T_{\psi_{R}}$-term, this time we use Lemma 2.8(ii), which implies that the symmetric $\mu_{*}$-part of the principal value vanishes:

$$
\begin{aligned}
\lim _{\delta \rightarrow 0} T_{\psi_{R}}^{\delta} & =-\tilde{I}_{1}\left[\tilde{\psi}_{R}\right](x)-\frac{1}{R}\left[\frac{d \psi}{d s}\left(\left|x^{\prime}\right| / R\right)\right]^{\prime} \cdot \int_{0<|z|<1} \eta(x, z)^{\prime} d \mu_{\#}(z)+(\cdots)(y) \\
& \leq C(\mu)\left(\frac{1}{R^{2}}\|\psi\|_{C^{2}}+\frac{1}{R}\|\psi\|_{C^{1}}\right)=o_{R}(1) .
\end{aligned}
$$

Thus when $\varepsilon^{\prime}, \varepsilon_{N}, \nu, R>0$ are fixed and we send $\delta \rightarrow 0$, we obtain

$$
\begin{aligned}
u(x)-v(y) \leq & f(x)-f(y)+o_{\nu}(1)+o_{R}(1) \\
& +\frac{1}{\varepsilon^{\prime 2}} \int_{|z|<1}\left|\eta(x, z)^{\prime}-\eta(y, z)^{\prime}\right|^{2} d \mu(z) \\
& +\frac{2}{\varepsilon^{\prime 2}} \int_{|z|<1}\left(x^{\prime}-y^{\prime}\right) \cdot\left(\eta(x, z)^{\prime}-\eta(y, z)^{\prime}\right) d \mu_{\#}(z) \\
& +\int_{|z| \geq 1}\{u(P(x, z))-v(P(y, z))-u(x)+v(y)\} d \mu(z) \\
& =f(x)-f(y)+o_{\nu}(1)+o_{R}(1)+\operatorname{Int}_{1}+\operatorname{Int}_{2}+\operatorname{Int}_{3}
\end{aligned}
$$

as $\nu \rightarrow 0$ and $R \rightarrow \infty$, where the $o_{R}(1)$ - and $o_{\nu}(1)$-terms are independent of the other parameters.

Step 3. Sending the parameters to their limits. We have seen that by (4.1), $\mid x_{N}-$ $y_{N} \mid \leq \bar{c} \varepsilon_{N}$, where $\bar{c}$ only depends on the infinite norm of $u$ and $v$. Moreover, $|x|,|y|<R$. Hence we can assume (taking subsequences) that $x, y$ converge as $\varepsilon_{N} \rightarrow 0$ to points, still denoted by $x, y$, such that $x_{N}=y_{N}$. 
Now, combining $\left(\mathrm{H}_{\eta}^{3}\right)$ and $\left(\mathrm{H}_{\eta}^{4}\right)$, we then obtain that in the limit,

$$
\lim _{\varepsilon_{N} \rightarrow 0}\left|\eta(x, z)^{\prime}-\eta(y, z)^{\prime}\right| \leq L_{\eta}|z|\left|x^{\prime}-y^{\prime}\right| \quad \text { for } \mu \text {-a.e. } z \text {. }
$$

By $\left(\mathrm{H}_{\eta}^{1}\right)$ and the integrability condition on $\mu_{\#}$, we can use the dominated convergence theorem in $\mathrm{Int}_{2}$. The argument is similar for $\mathrm{Int}_{1}$, now using the domination

$$
\left|\eta(x, z)^{\prime}-\eta(y, z)^{\prime}\right|^{2} \leq\left(2 c_{\eta}\right)^{2}|z|^{2} .
$$

The result is that

$$
\lim _{\varepsilon_{N} \rightarrow 0} \operatorname{Int}_{2}=0 \quad \text { and } \quad \lim _{\varepsilon_{N} \rightarrow 0} \operatorname{Int}_{1} \leq\left(2 c_{\eta}\right)^{2} \frac{\left|x^{\prime}-y^{\prime}\right|^{2}}{\varepsilon^{\prime 2}} \int_{|z|<1}|z|^{2} d \mu(z)=o_{\varepsilon^{\prime}}(1) .
$$

Next, since $|x-y| \rightarrow 0$ as $\varepsilon_{N}, \varepsilon^{\prime} \rightarrow 0$ here, we may assume that $x, y \rightarrow \bar{x} \in \Omega$ as $\varepsilon_{N}, \varepsilon^{\prime} \rightarrow 0$ by considering subsequences if necessary. Then, also using the continuity of $f$,

$$
\lim _{\varepsilon^{\prime} \rightarrow 0} \lim _{\varepsilon_{N} \rightarrow 0} \operatorname{Int}_{1} \leq 0 \quad \text { and } \quad \lim _{\varepsilon^{\prime} \rightarrow 0} \lim _{\varepsilon_{N} \rightarrow 0}|f(x)-f(y)|=0 .
$$

We then also pass to the limit as $\nu \rightarrow 0$, taking converging subsequences of $\bar{x}$ and calling the limit $\bar{x}$ again, to find that

$$
u(\bar{x})-v(\bar{x}) \leq 0+\limsup _{\nu \rightarrow 0} \limsup _{\varepsilon^{\prime} \rightarrow 0} \limsup _{\varepsilon_{N} \rightarrow 0}\left[\operatorname{Int}_{3}\right]+o_{R}(1) .
$$

Since the integrand is bounded and $\mu$ is finite on $\{|z| \geq 1\}$, we can use Fatou's Lemma and upper semicontinuity of $u-v$ to pass to the limit in the $\mathrm{Int}_{3}$-term. Since

$$
\lim _{\nu \rightarrow 0} \lim _{\varepsilon^{\prime} \rightarrow 0} \lim _{\varepsilon_{N} \rightarrow 0}(u(x)-v(y))=M+o_{R}(1)
$$

by (4.2), we then find that

$$
\begin{aligned}
& \limsup _{\nu \rightarrow 0} \limsup _{\varepsilon^{\prime} \rightarrow 0} \limsup _{\varepsilon_{N} \rightarrow 0}\left[\operatorname{Int}_{3}\right] \\
& \leq \int_{|z| \geq 1}\left\{u(P(\bar{x}, z))-v(P(\bar{x}, z))-\left(M+o_{R}(1)\right)\right\} d \mu(z) .
\end{aligned}
$$

Now since $u(P(\bar{x}, z))-v(P(\bar{x}, z)) \leq \sup _{\bar{\Omega}}(u-v)=M$,

$$
\limsup _{\nu \rightarrow 0} \limsup _{\varepsilon^{\prime} \rightarrow 0} \limsup _{\varepsilon_{N} \rightarrow 0}\left[\operatorname{Int}_{3}\right] \leq \int_{|z| \geq 1} o_{R}(1) d \mu(z)=o_{R}(1) .
$$

When $R \rightarrow \infty$ in (4.3), we get $M \leq 0$ and the proof is complete.

\section{Comparison in the CEnsored CASE. I}

In this section we give comparison and well-posedness results for the initial value problem (1.1) in the censored case (under assumption $\left(\mathrm{H}_{\eta}^{6}\right)$ ) when the measure $\mu$ is not too singular:

$\left(\mathrm{H}_{\mu}^{\prime}\right)$ The measure $\mu$ is a nonnegative Radon measure satisfying
(i) $\int_{\mathbb{R}^{N}}|z| \wedge 1 d \mu<\infty$ and
(ii) $\int_{\left\{z_{N}=a\right\}} d \mu=0$ for any $a<0$. 
In addition, we assume the existence of a "blow-up supersolution":

(U) There exists $R_{0}>0$ such that, for any $R>R_{0}$, there is a positive function $\mathcal{U}_{R} \in C^{2}(\Omega)$ such that

$$
-I\left[\mathcal{U}_{R}\right](x) \geq-K_{R} \quad \text { in } \quad\left\{x: 0<x_{N} \leq R\right\},
$$

for some $K_{R} \geq 0$, and

$$
\mathcal{U}_{R}(x) \geq \frac{1}{\omega_{R}\left(x_{N}\right)} \quad \text { in } \quad \Omega
$$

for some function $\omega_{R}$ which is nonnegative, continuous, strictly increasing in a neighbourhood of 0 , and satisfies $\omega(0)=0$.

Remark 5.1. See Appendix A for a discussion of this assumption. For example, in Remark A.4 we prove that (U) holds if

$$
\mu=\bar{\mu}+\sum_{i=1}^{M} c_{i} \delta_{x^{i}},
$$

where $c_{i} \in \mathbb{R}, x_{i} \in \Omega, \delta_{x^{i}}$ are delta measures supported at $\left\{x^{i}\right\}$, and

$$
\frac{d \bar{\mu}}{d z}=\frac{g(z)}{|z|^{N+\alpha}} \quad \text { where } \quad \alpha \in(0,1), 0 \leq g \in L^{\infty}(\mathbb{R}), \lim _{z \rightarrow 0} g(z)=g(0)>0 .
$$

This class of measures include the Lévy measures of the stable, tempered stable, and self-decomposable Lévy processes. Much more general examples are presented in Appendix $\mathrm{A}$

Theorem 5.2. Assume $\left(\mathrm{H}_{\mu}^{\prime}\right),\left(\mathrm{H}_{f}\right),\left(\mathrm{H}_{\eta}^{6}\right)$ and $(U)$ hold. Let $u$ be a bounded usc subsolution of (1.1) and $v$ be a bounded lsc supersolution of (1.1). Then $u \leq v$ in $\bar{\Omega}$.

As in the previous section, we have a well-posedness result for (1.1) by Theorems 5.2 and 3.2 .

Corollary 5.3. Under the assumptions of Theorem [5.2, there exists a unique viscosity solution of (1.1) depending continuously on $f$.

Proof of Theorem 5.2. We argue by contradiction assuming that $M:=\sup _{\bar{\Omega}}(u-$ $v)>0$. Take $R>R_{0}$ and $0<\kappa \ll 1$. Using $0<\varepsilon \ll 1$, we double the variables by introducing the quantities

$$
\begin{aligned}
& \phi(x, y)=\frac{|x-y|^{2}}{\varepsilon^{2}}+\kappa\left[\mathcal{U}_{R}(x)+\mathcal{U}_{R}(y)\right]+\psi_{R}(x)+\psi_{R}(y), \\
& \Phi(x, y)=u(x)-v(y)-\phi(x, y)
\end{aligned}
$$

where $\mathcal{U}_{R}$ is defined in $(\mathrm{U})$ and $\psi_{R}(x)=2\left(\|u\|_{\infty}+\|v\|_{\infty}\right) \psi\left(\frac{|x|}{R}\right)$ for an increasing function $\psi(s) \in C^{\infty}(0, \infty)$ which is 0 in $\left(0, \frac{1}{2}\right)$ and 1 in $(1, \infty)$.

For any $R, \kappa$ and $\varepsilon$, the function $\Phi$ achieves its maximum at $(\bar{x}, \bar{y})=\left(\bar{x}_{R, \kappa, \varepsilon}, \bar{y}_{R, \kappa, \varepsilon}\right)$ and, by the definition of $\mathcal{U}_{R}$ and $\psi_{R}$, we have

$$
\bar{x}_{N}, \bar{y}_{N} \geq \delta_{0}=\omega_{R}^{-1}\left(\frac{\kappa}{2\left(\|u\|_{\infty}+\|v\|_{\infty}\right)}\right) \quad \text { and } \quad|\bar{x}|,|\bar{y}| \leq R .
$$

For fixed $R$ and $\kappa$, a standard argument also show that

$$
\frac{|\bar{x}-\bar{y}|^{2}}{\varepsilon^{2}} \rightarrow 0 \quad \text { as } \quad \varepsilon \rightarrow 0 .
$$


By the estimates on $\bar{x}, \bar{y}$ and extracting subsequences if necessary, we can assume without loss of generality that $\bar{x}, \bar{y} \rightarrow X, u(\bar{x}) \rightarrow u(X)$, and $v(\bar{y}) \rightarrow v(X)$, where $X$ is a maximum point of $\Phi(x, x)=u(x)-v(x)-\phi(x, x)$. When we send $\kappa \rightarrow 0$ and then $R \rightarrow+\infty$, we have

$$
u(X)-v(X) \rightarrow M \quad \text { and } \quad \kappa \mathcal{U}_{R}(X)+\psi_{R}(X) \rightarrow 0 .
$$

Now we write the viscosity inequalities. Since $u-\phi(\cdot, \bar{y})$ has a global maximum at $\bar{x}$ and $v-(-\phi(\bar{x}, \cdot))$ has a global minimum at $\bar{y}$, by Definition 2.3

$$
\begin{aligned}
u(\bar{x})-I^{\delta}[u](\bar{x})-I_{\delta}[\phi(\cdot, \bar{y})](\bar{x}) & \leq f(\bar{x}) \quad \text { and } \\
v(\bar{y})-I^{\delta}[v](\bar{y})-I_{\delta}[-\phi(\bar{x}, \cdot)](\bar{y}) & \geq f(\bar{y}),
\end{aligned}
$$

for any $\delta>0$. With this in mind we see that

$$
\begin{aligned}
& M+o(1)=u(\bar{x})-v(\bar{y})-\phi(\bar{x}, \bar{y}) \\
& \leq I^{\delta}[u](\bar{x})-I^{\delta}[v](\bar{y})+I_{\delta}[\phi(\cdot, \bar{y})](\bar{x})-I_{\delta}[\phi(\bar{x}, \cdot)](\bar{y})+f(\bar{x})-f(\bar{y}) .
\end{aligned}
$$

In this inequality, we aim at first sending $\delta \rightarrow 0$ in order to get rid of the $\varepsilon$-depending $I_{\delta}[\phi]$-terms. In fact $I_{\delta}[\varphi] \rightarrow 0$ as $\delta \rightarrow 0$ by the Dominated Convergence Theorem since $|\eta(x, z)| \leq c_{\eta}|z|$, and hence for any $C^{1}$-function $\varphi$,

$$
\int_{\mathbb{R}^{N}}|\varphi(x+\eta(x, z))-\varphi(x)| 1_{|z|<\delta} d \mu(z) \leq c_{\eta}\|D \varphi\|_{L^{\infty}\left(\bar{B}\left(0, c_{\eta} \delta\right)\right)} \int_{\mathbb{R}^{N}} 1_{|z|<\delta}|z| d \mu(z) .
$$

Next we consider the $I^{\delta}$-terms. We restrict ourselves to a subsequence such that $\bar{x}_{N} \geq \bar{y}_{N}$ (if $\bar{x}_{N} \leq \bar{y}_{N}$, the argument is similar). Then

$$
\begin{aligned}
I^{\delta}[u](\bar{x})-I^{\delta}[v](\bar{y})= & \int_{-\bar{x}_{N}<z_{N}<-\bar{y}_{N}}[u(\bar{x}+z)-u(\bar{x})] 1_{|z|>\delta} d \mu(z) \\
& +\int_{-\bar{y}_{N}<z_{N}}[u(\bar{x}+z)-v(\bar{y}+z)-(u(\bar{x})-v(\bar{y}))] 1_{|z|>\delta} d \mu(z) \\
= & : I_{1}+I_{2} .
\end{aligned}
$$

For $I_{1}$, we have

$$
\left|I_{1}\right| \leq 2\|u\|_{\infty} \int_{|z|>\delta} 1_{\left\{-\bar{x}_{N}<z_{N}<-\bar{y}_{N}\right\}}(z) d \mu(z) .
$$

Keeping $\kappa$ and $R$ fixed and recalling (5.1), we see that this integral is independent of $\delta$ as soon as $\delta<\delta_{0}$. Furthermore, because of $\left(\mathrm{H}_{\mu}^{\prime}\right)(i i)$, the Dominated Convergence Theorem implies that

$$
I_{1} \rightarrow 0 \quad \text { as } \quad \varepsilon \rightarrow 0
$$

since $|\bar{x}-\bar{y}| \rightarrow 0$ as $\varepsilon \rightarrow 0$.

For $I_{2}$, we use the maximum point property for $\bar{x}, \bar{y}$,

$$
(u(\bar{x}+z)-v(\bar{y}+z))-(u(\bar{x})-v(\bar{y})) \leq \phi(\bar{x}+z, \bar{y}+z)-\phi(\bar{x}, \bar{y}),
$$

which after cancellation of the $\varepsilon$-terms leads to

$$
I_{2} \leq \kappa\left(I^{\delta}\left[\mathcal{U}_{R}\right](\bar{x})+I^{\delta}\left[\mathcal{U}_{R}\right](\bar{y})\right)+\left(I^{\delta}\left[\psi_{R}\right](\bar{x})+I^{\delta}\left[\psi_{R}\right](\bar{y})\right)
$$

Recalling (5.1) again and using the regularity of $\mathcal{U}_{R}$ and $\phi$, we can send $\delta \rightarrow 0$ and obtain

$$
\limsup _{\delta \rightarrow 0} I_{2} \leq \kappa\left(I\left[\mathcal{U}_{R}\right](\bar{x})+I\left[\mathcal{U}_{R}\right](\bar{y})\right)+\left(I\left[\psi_{R}\right](\bar{x})+I\left[\psi_{R}\right](\bar{y})\right)
$$


where the terms on the right-hand side are well defined by the properties of $\mathcal{U}_{R}$ and $\psi_{R}$.

Consider equation (5.2) again. Using all the previous estimates, we can send $\delta \rightarrow 0$ first and obtain using (U) for the $\mathcal{U}_{R}$-terms that

$$
M+o(1) \leq 2 K_{R} \kappa+\left(I\left[\psi_{R}\right](\bar{x})+I\left[\psi_{R}\right](\bar{y})\right)+(f(\bar{x})-f(\bar{y})) .
$$

In this inequality, we can first send $\varepsilon \rightarrow 0$, keeping $R$ and $\kappa$ fixed. Then $f(\bar{x})-$ $f(\bar{y}) \rightarrow 0$ as $\varepsilon \rightarrow 0$ since $f$ is uniformly continuous in $\bar{B}_{R}$, and we find that

$$
M+o(1) \leq 2 K_{R} \kappa+2 I\left[\psi_{R}\right](X) .
$$

We conclude the proof by first sending $\kappa \rightarrow 0$ and then $R \rightarrow+\infty$.

\section{Comparison Results in the CEnsored CASE. II}

In this section we give comparison and well-posedness results for the initial value problem (1.1) in the censored case (under assumption $\left(\mathrm{H}_{\eta}^{6}\right)$ ) when the measure $\mu$ is very singular. To this end we make the following assumption:

$\left(\mathrm{H}_{\mu}^{\prime \prime}\right)$ Hypothesis $\left(\mathrm{H}_{\mu}\right)$ holds with

$\mu_{*}(d z)=\frac{d z}{|z|^{N+\alpha}}, \int_{\mathbb{R}^{N}}\left(1 \wedge|z|^{\bar{\beta}}\right) \mu_{\#}(d z)<\infty, \int_{\left\{z_{N}=a\right\}} \mu_{\#}(d z)=0$ for any $a<0$,

for $\alpha \in(1,2)$ and $\bar{\beta}:=\alpha-1$.

This assumption is much more restrictive than $\left(\mathrm{H}_{\mu}\right)$, and the results of this section are not completely satisfactory. We had much difficulty in obtaining comparison results because on one hand it is not possible to get rid of the boundary and the boundary condition in such a general way as we did in the less singular case I. On the other hand many of the technical difficulties came from the way the $x$-depending domain of integration in $I$ interfered with the singularity of the measure and the boundary.

Our first result is the following:

Theorem 6.1. Assume $\left(\mathrm{H}_{f}\right),\left(\mathrm{H}_{\eta}^{6}\right)$, and $\left(\mathrm{H}_{\mu}^{\prime \prime}\right)$ hold.

(a) Let $u$ and $v$ be respectively a bounded usc subsolution and a bounded lsc supersolution of

$$
w(x)-I[w](x)=f(x) \text { in } \Omega,
$$

and let us also denote by $u$ and $v$ respectively their usc or lsc extensions to $\bar{\Omega} \overline{1}$ If

$$
u\left(x^{\prime}, x_{N}\right) \geq u\left(x^{\prime}, 0\right)+o\left(x_{N}^{\bar{\beta}}\right) \text { and } v\left(x^{\prime}, x_{N}\right) \leq v\left(x^{\prime}, 0\right)+o\left(x_{N}^{\bar{\beta}}\right) \text { as } x_{N} \rightarrow 0,
$$

uniformly in $x^{\prime}$ on compacts, then $u$ and $v$ are respectively a bounded usc subsolution and a bounded lsc supersolution of (1.1).

(b) If $u$ and $v$ are respectively a bounded usc subsolution and a bounded lsc supersolution of (1.1) satisfying (6.2), then

$$
u \leq v \quad \text { in } \bar{\Omega}
$$

in particular, there exists at most one solution of (1.1) in $C^{0, \beta}(\Omega)$ for $\beta>\bar{\beta}$.

\footnotetext{
${ }^{1}$ For any $x^{\prime} \in \mathbb{R}^{N-1}, \quad u\left(x^{\prime}, 0\right):=\limsup _{\left(y^{\prime}, y_{N}\right) \rightarrow\left(x^{\prime}, 0\right)} u\left(y^{\prime}, y_{N}\right)$ and $v\left(x^{\prime}, 0\right) \quad:=$ $\liminf \operatorname{s}_{\left(y^{\prime}, y_{N}\right) \rightarrow\left(x^{\prime}, 0\right)} v\left(y^{\prime}, y_{N}\right)$.
} 
Remark 6.2. The meaning of Theorem 6.1 (a) is that for sub- and supersolutions having a suitable regularity at the boundary, the Neumann boundary condition is already encoded in the equation/inequality holding in $\Omega$. Hence any solution of the equation in $\Omega$, with a suitable regularity on the boundary, will have an extension to $\bar{\Omega}$ which solves (1.1). In the setting of solutions, (6.2) is consistent with (1.5).

Unfortunately Theorem 6.1 (b) does not provide the full comparison result for semicontinuous sub- and supersolutions, and we do not know if this result is optimal or not. In view of Theorem 6.1 (b), it is clear that we need a companion existence result providing the existence of solutions satisfying (6.2) or belonging to $C^{0, \beta}(\Omega)$ for $\beta>\bar{\beta}$. We address this question after the proof of Theorem 6.1.

Proof. (a) We only prove the subsolution case since the supersolution case is analogous. Let $\phi$ be a smooth function and assume that $u-\phi$ has a maximum point $\bar{x}=\left(\bar{x}^{\prime}, 0\right) \in \partial \Omega$ in $\bar{B}\left(\bar{x}, c_{\eta} \delta\right) \cap \bar{\Omega}$. We may assume that the maximum is strict and global without any loss of generality.

Let $\theta(t)=t^{\bar{\beta}} \wedge 1$ for $t \geq 0$, and for $0<\kappa \ll 1$, we consider the function

$$
u(x)-\phi(x)+\kappa \theta\left(x_{N}\right) .
$$

This function has a global maximum point $x_{\kappa}$ near $\left(\bar{x}^{\prime}, 0\right)$, and we claim that $\left(x_{\kappa}\right)_{N}>0$. If $\left(x_{\kappa}\right)_{N}=0$, then $x_{\kappa}=\left(\bar{x}^{\prime}, 0\right)$, the strict global maximum point of $u-\phi$ on $\partial \Omega$. But then by (6.2),

$u\left(\bar{x}^{\prime}, 0\right)-\phi\left(\bar{x}^{\prime}, 0\right) \geq u\left(\bar{x}^{\prime}, a\right)-\phi\left(\bar{x}^{\prime}, a\right)+\kappa \theta(a) \geq u\left(\bar{x}^{\prime}, 0\right)-\phi\left(\bar{x}^{\prime}, 0\right)+o\left(a^{\bar{\beta}}\right)+\kappa \theta(a)$, which is a contradiction since $o\left(a^{\bar{\beta}}\right)+\kappa \theta(a)>0$ for $a$ small enough.

By Definition 2.3, we may write the viscosity subsolution inequality at $x_{\kappa}$ as

$$
u\left(x_{\kappa}\right)-\tilde{I}_{\delta}[\phi]\left(x_{\kappa}\right)-\gamma\left(x_{\kappa}\right) \cdot D \phi\left(x_{\kappa}\right)+\kappa I_{\delta}[\theta]\left(x_{\kappa}\right)-I^{\delta}[u]\left(x_{\kappa}\right) \leq f\left(x_{\kappa}\right),
$$

for (say) $0<\delta<1$, where $\gamma\left(x_{\kappa}\right) \mathrm{P} . \mathrm{V} \cdot \int_{|z|<\delta} \eta\left(x_{\kappa}, z\right) \mu(d z)$.

We first consider the term $\kappa I_{\delta}[\theta]\left(x_{\kappa}\right)$. By $\left(\mathrm{H}_{\mu}\right), \mu=\mu_{\#}+\mu_{*}$, and we may write $\kappa I_{\delta}[\theta]$ as a sum of an integral involving $\mu_{\#}$ and one involving $\mu_{*}$. The integral involving $\mu_{\#}$ is $O(\kappa)$ since $\theta$ is in $C^{0, \bar{\beta}}$ and $\left(\mathrm{H}_{\mu}^{\prime \prime}\right)$ holds, while the other integral (the $\mu_{*}$-integral) is given by

$$
\kappa \mathrm{P} . \mathrm{V} . \int_{\substack{|z| \leq \delta \\ x_{N}+z_{N} \geq 0}} \theta\left(x_{N}+z_{N}\right)-\theta\left(x_{N}\right) \frac{d z}{|z|^{N+\alpha}},
$$

where we have dropped the subscript $\kappa$ to simplify the notation. Since $\delta<1$ and $x_{N} \rightarrow 0$ as $\kappa \rightarrow 0$, we may assume that $0 \leq x_{N}+z_{N}<1$ for $|z| \leq \delta$, and hence that the principal value reduces to

$$
\kappa \mathrm{P} . \mathrm{V} . \int_{\substack{|z| \leq \delta \\ x_{N}+z_{N} \geq 0}}\left|x_{N}+z_{N}\right|^{\bar{\beta}}-\left|x_{N}\right|^{\bar{\beta}} \frac{d z}{|z|^{N+\alpha}} .
$$

By the computations of Lemma B.1 in the Appendix,

$$
-\mathrm{P} . \mathrm{V} . \int_{x_{N}+z_{N} \geq 0}\left|x_{N}+z_{N}\right|^{\bar{\beta}}-\left|x_{N}\right|^{\bar{\beta}} \frac{d z}{|z|^{N+\alpha}}=0
$$

for $x_{N}>0$. Writing

$$
\kappa \text { P.V. } \int_{\substack{|z| \leq \delta \\ x_{N}+z_{N} \geq 0}}(\cdots)=\kappa \text { P.V. } \int_{x_{N}+z_{N} \geq 0}(\cdots)-\kappa \int_{\substack{|z|>\delta \\ x_{N}+z_{N} \geq 0}}(\cdots)
$$


we conclude that for fixed $\delta$,

$$
\kappa \mathrm{P} . \mathrm{V} . \int_{\substack{|z| \leq \delta \\ x_{N}+z_{N} \geq 0}} \theta\left(x_{N}+z_{N}\right)-\theta\left(x_{N}\right) \frac{d z}{|z|^{N+\alpha}}=O(\kappa) .
$$

Finally, the $u,-\tilde{I}_{\delta}$,- and $I^{\delta}$-terms are uniformly bounded in $\kappa$ while $\gamma\left(x_{\kappa}\right) \rightarrow \infty$ since $\left(x_{\kappa}\right)_{N} \rightarrow 0$. We divide (6.3) by $\left|\gamma\left(x_{\kappa}\right)\right|$ and send $\kappa \rightarrow 0$. As in the proof of Theorem 3.2, the second part when $x \in \partial \Omega$ and $c=1$, the result is that all terms vanish except the $\gamma$-term, and we are left with the boundary condition

$$
\frac{\partial \phi}{\partial \mathbf{n}}(x) \leq 0 \text {. }
$$

(b) By linearity of the problem and part (a), the function $w=u-v$ is a subsolution of (1.1) with $f \equiv 0$, and we are done if we can prove that $w \leq 0$. To prove this, we consider the function

$$
\chi_{R, \nu}(x):=\psi\left(\left|x_{N}\right| / R\right)+\psi\left(\left|x^{\prime}\right| / R\right)-\nu \mathrm{d}\left(x_{N}\right),
$$

where $\psi$ and $\mathrm{d}$ are defined as in the proof of Theorem 4.1, replacing, in the case of $\psi, 2\left(\|u\|_{\infty}+\|v\|_{\infty}+1\right)$ by $2\|w\|_{\infty}+1$. The function $\chi_{R, \nu}$ is smooth, and easy computations show that $\chi_{R, \nu}$ is a supersolution of (1.1) with an $f \geq \varpi(R, \nu)$, where $\varpi(R, \nu) \rightarrow 0$ as $R \rightarrow \infty$ and $\nu \rightarrow 0$. At the boundary $\partial \Omega$,

$$
-\frac{\partial \chi_{R, \nu}}{\partial x_{N}}=0+\nu \cdot 1>0 .
$$

Because of the behavior of $\chi_{R, \nu}$ at infinity, the function $w-\chi_{R, \nu}$ achieves its maximum at some point $x$, and because of the behaviour of $\chi_{R, \nu}$ at the boundary, $x_{N}>0$. By Definition 2.3 we then have the following subsolution inequality:

$$
w(x)-\chi_{R, \nu}(x) \leq-\chi_{R, \nu}(x)+I\left[\chi_{R, \nu}\right](x)+I^{\delta}\left[u-\chi_{R, \nu}\right](x) \leq-\varpi(R, \nu)+0,
$$

where we have used that $I^{\delta}[w](\bar{x}) \leq 0$ at any maximum point $\bar{x}$ of the function $w$. Hence, for any $y \in \bar{\Omega}$,

$$
w(y)-\chi_{R, \nu}(y) \leq-\varpi(R, \nu),
$$

and part (b) follows from sending $R \rightarrow \infty$ and then $\nu \rightarrow 0$.

Now we turn to the existence of Hölder continuous solutions and we begin with a result in dimension 1 .

Theorem 6.3. Assume $N=1$ and that $\left(\mathrm{H}_{f}\right),\left(\mathrm{H}_{\eta}^{6}\right)$, and $\left(\mathrm{H}_{\mu}^{\prime \prime}\right)$ hold.

(a) Any bounded, uniformly continuous solution of (1.1) is in $C^{0, \beta}(\bar{\Omega})$ for some $\beta>\bar{\beta}$.

(b) There exists a solution of (1.1) in $C^{0, \beta}(\bar{\Omega})$ for some $\beta>\bar{\beta}$.

Proof. (a) Let $C>0$. To prove the Hölder regularity we consider

$$
\text { (6.4) } M=\sup _{[0,+\infty) \times[0,+\infty)}(u(x)-u(y)-\phi(x, y)) \quad \text { where } \quad \phi(x, y)=C|x-y|^{\beta} \text {, }
$$

and argue by contradiction assuming that $M>0$. The aim is to show that this is impossible for $C>0$ large enough.

In order to emphasize the main ideas and to avoid technicalities, we assume that this supremum is achieved at some point $(x, y)$ such that $x, y>0$. This assumption can be made rigorous by replacing $\phi$ by

$$
\tilde{\phi}(x, y)=\phi(x, y)+\psi_{R}(x, y)-d_{\nu}\left(x_{N}\right)-d_{\nu}\left(y_{N}\right)
$$


and arguing as in the proof of Theorem 4.1 where $\psi_{R}$ and $d_{\nu}$ are defined for $R, \nu>0$. The contribution from the new terms in the proof would be $o(1)$ as $\nu, R \rightarrow 0$.

Since $M>0$ we have $x \neq y$. We now assume that $x<y$, since the other case can be treated analogously. To simplify we redefine $\phi, \phi(z):=C|x-y+z|^{\beta}$. Note that this function is concave in the intervals $(-\infty, y-x)$ and $(y-x,+\infty)$, and that it is smooth in $(-\delta, \delta)$ for $\delta \leq y-x$ so that it can be used as a test function. By the maximum point property for $(x, y)$,

$$
u\left(x+z_{1}\right)-u\left(y+z_{2}\right)-C\left|x-y+\left(z_{1}-z_{2}\right)\right|^{\beta} \leq u(x)-u(y)-C|x-y|^{\beta},
$$

for $z_{1} \geq-x$ and $z_{2}>-y$, and hence

$$
\begin{aligned}
u(x+z)-u(y+z)-[u(x)-u(y)] & \leq 0 \quad \text { for } \quad z \geq-x(>-y) \\
u(x+z)-u(x) & \leq[\phi(z)-\phi(0)] \quad \text { for } \quad z \geq-x \\
u(y+z)-u(y) & \geq-[\phi(-z)-\phi(0)] \text { for } z \geq-y .
\end{aligned}
$$

Using the definition of viscosity solution and the symmetry of the measure $\mu_{*}$, for $\delta, \delta^{\prime}>0$ small enough, we have the inequalities

$$
-\left(I_{\delta}[\phi]+I^{\delta}[u]\right)(x)+u(x) \leq f(x) \text { and } \quad-\left(I_{\delta^{\prime}}[\phi]+I^{\delta^{\prime}}[u]\right)(y)+u(y) \geq f(y),
$$

which reduce here to

$$
\begin{aligned}
& -\int_{-x}^{-\delta}(u(x+z)-u(x)) d \mu(z)-\int_{-\delta}^{\delta}\left[\phi(z)-\phi(0)-\phi^{\prime}(0) z\right] d \mu(z) \\
& -\int_{-\delta}^{\delta} \phi^{\prime}(0) z d \mu(z)-\int_{\delta}^{+\infty}(u(x+z)-u(x)) d \mu(z)+u(x) \leq f(x), \\
& -\int_{-y}^{-\delta^{\prime}}(u(y+z)-u(y)) d \mu(z)+\int_{-\delta^{\prime}}^{\delta^{\prime}}\left[\phi(-z)-\phi(0)+\phi^{\prime}(0) z\right] d \mu(z) \\
& -\int_{-\delta^{\prime}}^{\delta^{\prime}} \phi^{\prime}(0) z d \mu(z)-\int_{\delta^{\prime}}^{+\infty}(u(y+z)-u(y)) d \mu(z)+u(y) \geq f(y) .
\end{aligned}
$$

In the proof below we will subtract these inequalities and the main difficulty of the proof will come from the term

$$
J:=-\int_{-y}^{-x}(u(y+z)-u(y)) d \mu(z)
$$

which is not a difference of terms from (6.8) and (6.9). Indeed the domain of integration $z \in(-y,-x)$ appears in inequality (6.9) but not in (6.8). Because of the singularity of $\mu$, if $x$ is close to 0 it is not obvious how to get an estimate for $J$ which is independent of $C$, or how to control this term with a bad sign by a term with a good sign. Therefore we have problems with this term if $x \rightarrow 0$ when $C \rightarrow+\infty$. For the $\mu_{\#}$-part of $J$ there is no problem. We can use (6.7) to see that $-\int_{-y}^{-x}(u(y+z)-u(y)) d \mu_{\#}(z) \leq \int_{-y}^{-x}[\phi(-z)-\phi(0)] d \mu_{\#}(z) \leq C \int_{-y}^{-x}|z|^{\beta} d \mu_{\#}(z)$, and we will see later that this term can be controlled since $|z|^{\beta}$ is $\mu_{\#}$-integrable.

First case. We first consider the case when $x \leq y-x$, or equivalently, $2 x \leq y$. In this case $J \geq 0$ and can be dropped from inequality (6.9). To see this we note that for $-y \leq z \leq-x$,

$$
2 x-y \leq x-y-z \leq x
$$


with $x \leq y-x$ and $2 x-y=-(y-x)+x \geq-(y-x)$, and hence by (6.7)

$$
u(y+z)-u(y) \geq-[\phi(-z)-\phi(0)]=|x-y|^{\beta}-|x-y-z|^{\beta} \geq 0 .
$$

In this first case, we choose $\delta=x$ and $\delta^{\prime}=y-x$ and subtract the viscosity inequalities (6.8) and (6.9). After some computations using (6.6), (6.7), and (6.10), and dropping the $J$-term, we are led to the inequality

$$
\begin{aligned}
& -\int_{-x}^{y-x}[\phi(z)+\phi(-z)-2 \phi(0)] d \mu(z) \\
& -\int_{y-x}^{+\infty}((u(x+z)-u(y+z))-(u(x)-u(y))) d \mu(z)+u(x)-u(y) \leq f(x)-f(y) .
\end{aligned}
$$

Some easy computations then show that the first integral equals

$$
-C(y-x)^{\beta-\alpha} \int_{-\frac{x}{y-x}}^{1}\left[|1+z|^{\beta}+|1-z|^{\beta}-2\right] \frac{d z}{|z|^{1+\alpha}}+O(C),
$$

where the $O(C)$-term comes from the $\mu_{\#}$ part of the measure since the integrand can be estimated by $2|z|^{\beta}$ which is integrable on, say, $(-1,1)$. The second integral is nonpositive by (6.5) and can be dropped because of the "-" in front.

Finally, since $f$ is bounded and $u(x)-u(y) \geq 0$ (by assumption), we obtain

$$
-C(y-x)^{\beta-\alpha} \int_{-\frac{x}{y-x}}^{1}\left[|1+z|^{\beta}+|1-z|^{\beta}-2\right] \frac{d z}{|z|^{1+\alpha}} \leq 2\|f\|_{\infty}+O(C) .
$$

In order to conclude, we use that $M=u(x)-u(y)-C|x-y|^{\beta}>0$ (by assumption) and $\beta \leq 1 \leq \alpha$ to find that

$$
|x-y| \leq\left(\frac{2\|u\|_{\infty}}{C}\right)^{1 / \beta} \text { and } C(y-x)^{\beta-\alpha} \geq K C^{\zeta},
$$

where $\zeta:=1+(\alpha-\beta) \beta^{-1}>1$ and $K=\left(2\|u\|_{\infty}\right)^{\frac{\beta-\alpha}{\beta}}$. Then we note that

$-\int_{-\frac{x}{y-x}}^{1}\left[|1+z|^{\beta}+|1-z|^{\beta}-2\right] \frac{d z}{|z|^{1+\alpha}} \geq-\int_{0}^{1}\left[|1+z|^{\beta}+|1-z|^{\beta}-2\right] \frac{d z}{|z|^{1+\alpha}}>0$,

since $z \mapsto|1+z|^{\beta}$ is strictly concave on $(-1,1)$. From inequality (6.11) we then find that

$$
\tilde{K} C^{\zeta} \leq 2\|f\|_{\infty}+O(C),
$$

which cannot hold for $C$ large enough and we have a contradiction in the first case.

Second case. We next consider the case when $x>y-x$, or equivalently, $2 x>y$. In this case we choose $\delta=\delta^{\prime}=y-x$, subtract viscosity inequalities (6.8) and (6.9), and use (6.7) to see that

$$
\begin{aligned}
& -\int_{-y}^{-x}[\phi(-z)-\phi(0)] d \mu(z)-\int_{-(y-x)}^{y-x}[\phi(z)+\phi(-z)-2 \phi(0)] d \mu(z) \\
& -\int_{y-x}^{+\infty}((u(x+z)-u(y+z))-(u(x)-u(y))) d \mu(z)+u(x)-u(y) \leq f(x)-f(y) .
\end{aligned}
$$

Arguing as in the first case, we can drop all $u$-terms and are led to an inequality of the form

$$
-C(y-x)^{\beta-\alpha}(B(a)+G) \leq 2\|f\|_{\infty}+O(C),
$$


where

$$
\begin{aligned}
B(a) & =\int_{-a-1}^{-a}\left(|1+z|^{\beta}-1\right) \frac{d z}{|z|^{N+\alpha}}, \\
G & =\int_{-1}^{1}\left(|1+z|^{\beta}+|1-z|^{\beta}-2\right) \frac{d z}{|z|^{N+\alpha}},
\end{aligned}
$$

with $a=x /(y-x)>1$. A technical computation (Corollary B.3 in the appendix) then shows that $B(a)+G \leq-\kappa<0$ for some $\beta>\bar{\beta}$ and we can conclude the argument as in the first case. The proof of (a) is complete.

Note the important estimate, valid in both cases: there exist $k_{1}, k_{2}>0$ such that

$$
I[u](x)-I[u](y) \leq-k_{1} C|x-y|^{\beta-\alpha}+k_{2}(1+C),
$$

where the 1 comes from the localization terms. This formal estimate should be interpreted in the viscosity sense and with the above choice(s) of test function and parameters $\delta$ and $\delta^{\prime}$; cf. e.g. (6.11).

(b) To show the existence of solutions with a suitable regularity property, we follow the so-called "Sirtaki method in 4 steps". We just give a sketch the proof which is an easy adaptation of the above arguments.

We start by building a suitable approximate problem. We approximate the Lévy measure $\mu$ by bounded measures $\mu_{n}=\mu 1_{|z|>1 / n}$ for $n \geq 1$ and denote the associated nonlocal term by $I_{n}$. Then we introduce a truncation of the nonlocal term and add an additional viscosity term. The result is the approximate equation

$$
-\epsilon u_{x x}-T_{R}\left(I_{n}[u]\right)+u=f \quad \text { in } \Omega,
$$

where $T_{R}(s):=\max (\min (s, R),-R), R, \epsilon>0$.

1. For fixed $\epsilon, n, R$, since the $T_{R}$-term and the measure $\mu_{n}$ are bounded, this equation can easily be solved by classical viscosity solutions' methods (Perron's method and comparison result). This provides us with a continuous solution which is bounded and we even have $\|u\|_{\infty} \leq\|f\|_{\infty}$.

Moreover, in order to obtain the $C^{0, \beta}$-regularity and $C^{0, \beta}$-bounds, we consider (6.4) and follow the arguments in the first part of this proof. After subtracting the viscosity sub- and supersolution inequalities, we formally obtain

$$
\begin{aligned}
-\epsilon\left[u_{x x}(x)-u_{x x}(y)\right]-\left[T_{R}\left(I_{n}[u]\right)(x)-\right. & \left.T_{R}\left(I_{n}[u]\right)(y)\right] \\
& +u(x)-u(y) \leq f(x)-f(y) .
\end{aligned}
$$

For the second derivatives, we have an analogue estimate to (6.12), namely there exists $k_{1}^{\prime}, k_{2}^{\prime}>0$ such that

$$
u_{x x}(x)-u_{x x}(y) \leq-k_{1}^{\prime} C|x-y|^{\beta-2}+k_{2}^{\prime} .
$$

Note that to give meaning to this formal estimate, we must consider instead of $u_{x x}$ the sub- and superjets of the theorem of sums; cf. e.g. [6]. Now consider (6.13) with fixed $R, \varepsilon>0$. Since the $T_{R}$-terms are bounded, we can rewrite it as

$$
-\epsilon\left[u_{x x}(x)-u_{x x}(y)\right] \leq 2 R+2\left(\|u\|_{\infty}+\|f\|_{\infty}\right),
$$

and use (6.14) to find that the inequality cannot hold for $C$ large enough. This implies that the solution $\left\{u^{n, R, \epsilon}\right\}$ is at least $C^{0, \beta}$ by the arguments of the regularity proof above. 
2. The above argument also shows that, for fixed $\epsilon$, the $C^{0, \beta}$-bounds for the $\left\{u^{n, R, \epsilon}\right\}$ are uniform in $n$ since they depend only on $R$ through the $T_{R}$-term. This allows us to pass to the limit $n \rightarrow+\infty$ and get a solution $u^{R, \epsilon}:=\lim _{n \rightarrow+\infty} u^{n, R, \epsilon}$ of the limiting equation enjoying the same $C^{0, \beta}$-bound. This solution satisfies the truncated viscous equation with $\mu_{n}$ replaced by the singular measure $\mu$.

3. Next, we repeat the proof of the $C^{0, \beta}$-bound for the truncated viscous equation: Estimate (6.12) together with the fact that $T_{R}$ is an increasing and a 1Lipschitz continuous function implies that

$$
T_{R}(I[u](x))-T_{R}(I[u](y)) \leq k_{2},
$$

at least for $C$ large enough. Rewriting the analogue of (6.13) as

$$
-\epsilon\left[u_{x x}(x)-u_{x x}(y)\right] \leq\left[T_{R}(I[u])(x)-T_{R}(I[u])(y)\right]+2\left(\|u\|_{\infty}+\|f\|_{\infty}\right),
$$

this new estimate on the difference of the truncated terms shows that the $C^{0, \beta}$ bound which is obtained in Step 1 is independent of $R$ and we can let $R \rightarrow+\infty$. The result is that the limit $u^{\varepsilon}:=\lim _{R \rightarrow \infty} u^{R, \varepsilon}$ is a $C^{0, \beta}$-solution of the nontruncated viscous equation

$$
-I[u]-\epsilon u_{x x}+u=f \quad \text { in } \Omega .
$$

4. Finally we come back again to the proof of the $C^{0, \beta}$-bound, but this time the main role is played by the nonlocal term via estimate (6.12). Indeed we rewrite the analogue of $(\underline{6.13})$ as

$$
-[I[u](x)-I[u](y)] \leq \epsilon\left[u_{x x}(x)-u_{x x}(y)\right]+2\left(\|u\|_{\infty}+\|f\|_{\infty}\right),
$$

and remark that, since the $u_{x x}$-terms satisfy (6.14), the $\epsilon$-term in (6.13) can be estimated by $\epsilon k_{2}^{\prime}$. Using (6.12), we again obtain a contradiction for large enough $C$. The argument is the same as in Step 3 with the roles of the local and nonlocal terms exchanged. This also explains the terminology "Sirtaki's method", since Sirtaki is a dance where we exchange the roles of the two feet and here we exchange the role of the $\epsilon u_{x x^{-}}$and $I[u]$-terms. To conclude the argument, we have found that the $C^{0, \beta}$-bound is independent of $\epsilon$, and we pass to the limit as $\epsilon \rightarrow 0$. We get a solution $u$ of the original problem belonging to $C^{0, \beta}$. Since this solution is unique, it is the solution we are looking for.

Now we turn to the case when $N \geq 2$. Unfortunately we require far more retrictive assumptions on $f$.

Theorem 6.4. Assume $N \geq 2$, that $\left(\mathrm{H}_{f}\right),\left(\mathrm{H}_{\eta}^{6}\right)$, and $\left(\mathrm{H}_{\mu}^{\prime \prime}\right)$ hold, and that $f\left(\ldots, x_{N}\right)$ is in $W^{2, \infty}\left(\mathbb{R}^{N-1}\right)$ for any $x_{N}>0$ with uniformly bounded $W^{2, \infty}$-norms.

(a) Any bounded, uniformly continuous solution of (1.1) is in $C^{0, \beta}(\bar{\Omega})$ for some $\beta>\bar{\beta}$.

(b) There exists a solution of (1.1) in $C^{0, \beta}(\bar{\Omega})$ for some $\beta>\bar{\beta}$.

Proof. We are not going to provide the full proof since it is rather long and tedious and is mostly based on two ingredients which we have already seen. But we remark that an easy consequence of the the comparison result and linearity of the problem is that $u$ inherits the regularity of $f$. That is to say, there exists a constant $K>0$ such that, for any $x^{\prime}, z^{\prime} \in \mathbb{R}^{N-1}$ and $x_{N}>0$,

$$
-K\left|z^{\prime}\right|^{2} \leq u\left(x^{\prime}+z^{\prime}, x_{N}\right)+u\left(x^{\prime}-z^{\prime}, x_{N}\right)-2 u\left(x^{\prime}+z^{\prime}, x_{N}\right) \leq K\left|z^{\prime}\right|^{2} .
$$


Then we repeat the 1 -d proof essentially considering

$$
\sup _{[0,+\infty) \times[0,+\infty)}\left(u\left(x^{\prime}, x_{N}\right)-u\left(x^{\prime}, y_{N}\right)-C\left|x_{N}-y_{N}\right|^{\beta}\right) .
$$

Of course, a doubling of variables in $x^{\prime}$ is necessary to take care of the singularity of the measure, but using the $W^{2, \infty}$ property in $x^{\prime}$, we can go back to the 1 -d computations without any difficulty. Let us just mention the key decomposition we use here. We rewrite the integrals with respect to $\mu_{*}$, first replacing the integrands by

$$
u\left(x^{\prime}+z^{\prime}, x_{N}+z_{N}\right)+u\left(x^{\prime}-z^{\prime}, x_{N}-z_{N}\right)-2 u\left(x^{\prime}, x_{N}\right),
$$

and then by

$$
\Delta_{z^{\prime}}^{2} u\left(x^{\prime}, x_{N}+z_{N}\right)+\Delta_{z^{\prime}}^{2} u\left(x^{\prime}, x_{N}-z_{N}\right)+2 \Delta_{z_{N}}^{2} u\left(x^{\prime}, x_{N}\right),
$$

where

$$
\begin{aligned}
\Delta_{z^{\prime}}^{2} u\left(x^{\prime}, x_{N}\right) & :=\frac{1}{2}\left(u\left(x^{\prime}+z^{\prime}, x_{N}\right)+u\left(x^{\prime}+z^{\prime}, x_{N}\right)-2 u\left(x^{\prime}, x_{N}\right)\right), \\
\Delta_{z_{N}}^{2} u\left(x^{\prime}, x_{N}\right) & :=\frac{1}{2}\left(u\left(x^{\prime}, x_{N}+z_{N}\right)+u\left(x^{\prime}, x_{N}-z_{N}\right)-2 u\left(x^{\prime}, x_{N}\right)\right) .
\end{aligned}
$$

These expressions are not equal pointwise of course, but they give the same integrals because of the symmetry of $\mu_{*}$. We deal with the $\Delta_{z^{\prime}}^{2}$-terms using (6.15), and the $\Delta_{z_{N}}^{2}$-term is treated as in the one dimensional case. Also note that we use a decomposition of $\Omega$ into sets like $\mathbb{R}^{N-1} \times\left\{z_{N}: a \leq z_{N} \leq b\right\}$, for $a, b>0$, following the 1-d proof.

Finally, concerning the nonsymetric part $\mu_{\#}$, we use as usual the fact that it is a controlled term since it is less singular.

The existence is proved as in the proof of Theorem 6.3

Remark 6.5. The regularity results of the $N=1$ and $N \geq 2$ cases are different. In the first case, the result is purely elliptic and we gain regularity. In the second case, the result is elliptic in the $x_{N}$-direction, while in the other directions we just use a preservation of regularity argument. It is an open problem to find an elliptic argument also in the $x^{\prime}$-directions.

\section{The LIMIT AS $\alpha \rightarrow 2^{-}$}

In this section we prove that all the Neumann models we consider converge to the same local Neumann problem as $\alpha \rightarrow 2^{-}$, provided that the nonlocal operators include the normalisation constant $(2-\alpha)$. To be more precise, we consider the following problem:

$$
\left\{\begin{aligned}
-(2-\alpha) \int_{\mathbb{R}^{N}} u_{\alpha}(x+\eta(x, z))-u_{\alpha}(x) d \mu_{\alpha}+u_{\alpha}(x) & =f(x) & & \text { in } \Omega, \\
\frac{\partial u}{\partial \mathbf{n}} & =0 & & \text { in } \partial \Omega,
\end{aligned}\right.
$$

where $\alpha \in(0,2), \eta$ depends on the Neumann model we consider, and

$$
\frac{d \mu_{\alpha}}{d z}=\frac{g(z)}{|z|^{N+\alpha}},
$$

where $g$ is nonnegative, continuous and bounded in $\mathbb{R}^{N}, g(0)>0$ and $g \in C^{1}(B)$ for some ball $B$ around 0 . 
We prove below that the solution of (7.1) converges to the solution of the following local problem:

$$
\left\{\begin{aligned}
-a \Delta u-b \cdot D u+u & =f & & \text { in } \Omega \\
\frac{\partial u}{\partial \mathbf{n}} & =0 & & \text { in } \partial \Omega,
\end{aligned}\right.
$$

where

$$
a:=g(0) \frac{\left|S^{N-1}\right|}{N} \quad \text { and } \quad b:=D g(0) \frac{\left|S^{N-1}\right|}{N} .
$$

In this section $\left|S^{N-1}\right|$ denotes the measure of the unit sphere in $\mathbb{R}^{N}$ and $\operatorname{Id}_{N}$ the $N \times N$ identity matrix.

Theorem 7.1. Assume $\left(\mathrm{H}_{\eta}^{i}\right), i=0, \ldots, 4$, hold and let $u_{\alpha}$ be the solution of (7.1) for $\alpha \in(0,2)$. Then, as $\alpha \rightarrow 2^{-}, u_{\alpha}$ converges locally uniformly to the unique solution $u$ of (7.2).

Before providing the proof, we introduce the following sequences of measures:

$$
\begin{aligned}
\left(d \nu_{\alpha}^{1}\right)_{i, j} & =(2-\alpha) z_{i} z_{j} \frac{g(z)}{|z|^{N+\alpha}} d z \\
d \nu_{\alpha}^{2} & =(2-\alpha) z \frac{g(z)-g(0)}{|z|^{N+\alpha}} d z \\
\left(d \nu_{\alpha, y}^{3}\right)_{i, j} & =(2-\alpha) \eta(y, z)_{i} \eta(y, z)_{j} \frac{g(z)}{|z|^{N+\alpha}} d z, \\
d \nu_{\alpha, y}^{4} & =(2-\alpha) \eta(y, z) \frac{g(z)-g(0)}{|z|^{N+\alpha}} d z,
\end{aligned}
$$

where $\eta(y, z)_{i}$ denotes the $i$-th component of the vector $\eta(y, z)$. Note that $\nu_{\alpha}^{1}$ and $\nu_{\alpha, y}^{3}$ are matrix measures while $\nu_{\alpha}^{2}$ and $\nu_{\alpha, y}^{4}$ are vector measures. The localization phenomenon occuring as $\alpha \rightarrow 2$ is reflected in the following lemma:

\section{Lemma 7.2.}

(a) As $\alpha \rightarrow 2^{-}, \nu_{\alpha}^{1} \rightarrow a \delta_{0} \operatorname{Id}_{N}$ and $\nu_{\alpha}^{2} \rightarrow b \delta_{0}$ in the sense of measures.

(b) For any sequence $\alpha_{k} \rightarrow 2$ and $y_{k} \rightarrow x \in \partial \Omega$, there exist two vector functions $\bar{a}(x), \bar{b}(x) \in \mathbb{R}^{N}$ satisfying

$$
\frac{1}{2} a \leq \bar{a}_{i}(x) \leq \Lambda \quad \text { and } \quad\left|\bar{b}_{i}(x)\right| \leq \Lambda \quad \text { for some } \Lambda=\Lambda(g, \eta)<\infty,
$$

such that, at least along a subsequence,

$$
\nu_{\alpha_{k}, y_{k}}^{3} \rightarrow \operatorname{diag}(\bar{a}(x)) \delta_{0}, \quad \nu_{\alpha_{k}, y_{k}}^{4} \rightarrow \bar{b}(x) \delta_{0},
$$

where $\operatorname{diag}(\bar{a}(x))$ is the diagonal matrix with diagonal coefficients $\bar{a}_{i}(x)$.

Proof. If $\delta \in(0,1)$ is fixed, we notice first that, for any $K>1$,

$$
0 \leq(2-\alpha) \int_{\delta<|z|<K}|z|^{2} \frac{g(z) d z}{|z|^{N+\alpha}} \leq\|g\|_{\infty}\left(\delta^{2-\alpha}-K^{2-\alpha}\right) \rightarrow 0 \text { as } \alpha \rightarrow 2^{-},
$$

so that the only possible limit in the sense of measure is supported in $\{0\}$. Similar calculations show that the same is true for all the measures $\nu^{i}, i=2, \ldots, 4$.

Next we remark that we may replace $g(z)$ by $g(0)$ in $\nu_{\alpha}^{1}, \nu_{\alpha, y}^{3}$ and $g(z)-g(0)$ by $(D g(0), z)$ in $\nu_{\alpha}^{2}, \nu_{\alpha, y}^{4}$ without changing the limits as $\alpha \rightarrow 2^{-}$. Indeed, the errors 
introduced can be expressed as uniformly bounded measures multiplied by $(2-\alpha)$ and will therefore tend to 0 in the limit. For example, in the case of $\nu_{\alpha}^{1}$, we have

$$
\begin{aligned}
& \left|(2-\alpha) \int_{|z|<\delta} z_{i} z_{j} \frac{g(z)-g(0)}{|z|^{N+\alpha}} d z\right| \\
& \leq C_{g}(2-\alpha) \int_{|z|<\delta} \frac{|z|^{3}}{|z|^{N+\alpha}} d z \leq C_{g}(2-\alpha) \frac{\delta^{3-\alpha}}{3-\alpha} \rightarrow 0 \quad \text { as } \alpha \rightarrow 2^{-},
\end{aligned}
$$

for $C_{g}=\|D g\|_{L^{\infty}(\bar{B}(0, \delta))}$. Similar arguments can be used for $\nu_{\alpha}^{2}, \nu_{\alpha, y}^{3}$ and $\nu_{\alpha, y}^{4}$.

Taking into account these reductions, we first examine $\nu_{\alpha}^{1}$. By symmetry

$$
(2-\alpha) \int_{|z|<\delta} z_{i} z_{j} \frac{g(0)}{|z|^{N+\alpha}} d z=0
$$

for $i \neq j$, while for $i=j$,

$$
\begin{aligned}
g(0)(2-\alpha) \int_{|z|<\delta} z_{i}^{2} \frac{d z}{|z|^{N+\alpha}} & =g(0) \frac{\left|S^{N-1}\right|}{N}(2-\alpha) \int_{r=0}^{\delta} \frac{r^{2+N-1}}{r^{N+\alpha}} d r \\
& =g(0) \frac{\left|S^{N-1}\right|}{N} \delta^{2-\alpha} \longrightarrow a \quad \text { as } \alpha \rightarrow 2^{-}
\end{aligned}
$$

This means that the measures $\left\{\nu_{\alpha}^{1}\right\}$ concentrate to a delta mass $\delta_{0}$ multiplied by the diagonal matrix $a \operatorname{Id}_{N}$.

For $\nu_{\alpha}^{2}$, by using similar arguments, we have

$$
\begin{aligned}
(2-\alpha) \int_{|z|<\delta} z_{i} \frac{(z, D g(0))}{|z|^{N+\alpha}} d z & =\sum_{j=1}^{N} \frac{\partial g}{\partial x_{j}}(0)(2-\alpha) \int_{|z|<\delta} z_{i} z_{j} \frac{d z}{|z|^{N+\alpha}} \\
& =\frac{\partial g}{\partial x_{i}}(0)(2-\alpha) \int_{|z|<\delta} z_{i}^{2} \frac{d z}{|z|^{N+\alpha}} \\
& \longrightarrow \frac{\partial g}{\partial x_{i}}(0) \frac{\left|S^{N-1}\right|}{N} \text { as } \alpha \rightarrow 2^{-} .
\end{aligned}
$$

Hence, by the definition of $b, \nu_{\alpha}^{2}$ concentrates to $b \delta_{0}$.

We now come to the measure $\nu^{3}$ which is more complex to analyze due to the presence of the perturbation $\eta\left(y_{k}, z\right)$. We first note that by $\left(\mathrm{H}_{\eta}^{2}\right)$, it follows that

$$
\int_{|z|<\delta} \eta\left(y_{k}, z\right)_{i} \eta\left(y_{k}, z\right)_{j} \frac{g(0) d z}{|z|^{N+\alpha}}=0 \quad \text { for } \quad i \neq j .
$$

Indeed, since $i \neq j$, either $i \neq N$ or $j \neq N$ and, for example, in the first case, we can use a change of variable with $\sigma_{i}$ to prove the claim. Then by $\left(\mathrm{H}_{\eta}^{1}\right)\left|\eta\left(y_{k}, z\right)\right| \leq c_{\eta}|z|$, and we have for $1 \leq i \leq N$

$$
\begin{aligned}
0 & \leq(2-\alpha) \int_{|z|<\delta} \eta\left(y_{k}, z\right)_{i}^{2} \frac{g(0) d z}{|z|^{N+\alpha}} d z \\
& \leq c_{\eta}^{2}(2-\alpha) \int_{|z|<\delta}|z|^{2} \frac{g(0) d z}{|z|^{N+\alpha}} d z \leq c_{\eta}^{2} g(0)\left|S^{N-1}\right| .
\end{aligned}
$$

So, the total mass of $\nu^{3}$ is bounded and, by the same arguments as above, it is clear that the support of $\nu^{3}$ shrinks to $\{0\}$ (or the empty set). We split the integral over 
$\{|z|<\delta\}$ as follows:

$$
(2-\alpha) \int_{|z|<\delta} \eta\left(y_{k}, z\right)_{i}^{2} \frac{g(0) d z}{|z|^{N+\alpha}}=\int_{\substack{|z|<\delta \\ z_{N}>-y_{k, N}}}(\cdots)+\int_{\substack{|z|<\delta \\ z_{N} \leq-y_{k, N}}}(\cdots)=\left(A_{i}\right)+\left(B_{i}\right) .
$$

Since $\eta_{i}\left(y_{k}, z\right)=z$ when $z_{N}>-y_{k, N}$ and $y_{k, N} \rightarrow x_{N}=0$,

$$
\begin{aligned}
\left(A_{i}\right) & =(2-\alpha) \int_{\substack{z_{N}>-y_{k, N} \\
|z|<\delta}} z_{i}^{2} \frac{g(0) d z}{|z|^{N+\alpha}} \\
& =(2-\alpha) \int_{\substack{|z|<\delta \\
z_{N}>0}} z_{i}^{2} \frac{g(0) d z}{|z|^{N+\alpha}}+o_{y_{k, N}}(1) \rightarrow \frac{1}{2} a .
\end{aligned}
$$

The other integral has a sign and can take different values according to the structure of the jumps, but in all cases we see that the weak limit of $\nu^{3}$ can be written as $\bar{a}(x) \delta_{0}$, where $\bar{a}(x)$ satisfies $\frac{a}{2} \leq \bar{a}_{i}(x) \leq \Lambda$.

The measure $\nu^{4}$ is treated similarly: the total mass can be bounded by

$$
\begin{aligned}
(2-\alpha) \int_{|z|<\delta}|\eta(y, z)| \frac{|(D g(0), z)|}{|z|^{N+\alpha}} d z & \leq c_{\eta}|D g(0)|(2-\alpha) \int_{|z|<\delta}|z|^{2} \frac{d z}{|z|^{N+\alpha}} \\
& =c_{\eta}\left|D g(0) \| S^{N-1}\right| \delta^{2-\alpha},
\end{aligned}
$$

so that, up to a subsequence, there indeed exists a vector function $\bar{b}$ such that $\nu_{\alpha_{n}, y_{n}}^{4} \rightarrow \bar{b} \delta_{0}$ in the sense of measures, with $\|\bar{b}\|_{\infty} \leq c_{\eta}\left|D g(0) \| S^{N-1}\right|$. The result then holds with $\Lambda:=\left|S^{N-1}\right| c_{\eta} \max \{|D g(0)|, g(0)\}$.

Remark 7.3. Note that in the censored case, $\bar{a}(x) \equiv a / 2$ since the jumps below level $-y_{N}$ are censored, while $\bar{a}(x)=a$ by symmetry when the jumps are mirror reflected. Under our general hypotheses, different structures of the jumps (i.e. different $\eta$ 's) lead to different $\bar{a}$ 's which could in principle depend on $x$ and the sequences $\alpha_{k}, y_{k}$. We will overcome this difficulty by using the extremal Pucci operator associated to $\bar{a}(x)$ : for any symmetric $N \times N$ matrix $A$ with eigenvalues $\left(\lambda_{i}\right)$ we define

$$
\mathcal{M}^{+}(A):=\frac{a}{2} \sum_{\lambda_{i}<0} \lambda_{i}+\Lambda \sum_{\lambda_{i}>0} \lambda_{i}
$$

Proposition 7.4. Let us define the half-relaxed limits as $\alpha \rightarrow 2^{-}$:

$$
\bar{u}(x):=\limsup _{\alpha \rightarrow 2, y \rightarrow x} u_{\alpha}(y) \quad \text { and } \quad \underline{u}(x):=\liminf _{\alpha \rightarrow 2, y \rightarrow x} u_{\alpha}(y) .
$$

Under the assumptions of Theorem 17.1, $\bar{u}$ is a viscosity subsolution of (7.2), and $\underline{u}$ is a viscosity supersolution of (7.2).

Proof. The proofs for $\bar{u}$ and $\underline{u}$ are similar, therefore we only provide it for $\bar{u}$. We have to check that $\bar{u}$ satisfies the viscosity subsolution inequalities for the Neumann problem (17.2) at any point $x \in \bar{\Omega}$. There are two separate cases to check, (i) when $x \in \Omega$ and (ii) when $x \in \partial \Omega$.

Step 1. Case (i) where $x \in \Omega$, that is, $x_{N}>0$. Let $\phi$ be a smooth function and assume that $x$ is a strict local maximum point of $u-\phi$. By standard arguments there exists a sequence $\left(y_{\alpha}\right)_{\alpha}$ of local maximum points of $u_{\alpha}-\phi$ such that $y_{\alpha} \rightarrow x$ 
as $\alpha \rightarrow 2^{-}$. Moreover, since $x_{N}>0$, by taking $\alpha$ close to 2 , we can assume that $y_{\alpha, N}>\delta$ for some small $\delta>0$. By the subsolution inequality for $u_{\alpha}$ at $y_{\alpha}$,

$$
\begin{array}{r}
-(2-\alpha) \int_{|z|<\delta} \phi\left(y_{\alpha}+z\right)-\phi\left(y_{\alpha}\right)-D \phi\left(y_{\alpha}\right) \cdot z d \mu_{\alpha}-(2-\alpha) \int_{|z|<\delta} D \phi\left(y_{\alpha}\right) \cdot z d \mu_{\alpha} \\
-(2-\alpha) \int_{|z| \geq \delta} u_{\alpha}\left(P\left(y_{\alpha}, z\right)\right)-u_{\alpha}\left(y_{\alpha}\right) d \mu_{\alpha}+u_{\alpha}\left(y_{\alpha}\right) \leq f\left(y_{\alpha}\right) .
\end{array}
$$

We recall that the second integral of the left-hand side is well defined; see the remark after Lemma 2.7.

We denote the three integral terms by $I_{1}, I_{2}$, and $I_{3}$. Then

$$
\begin{aligned}
I_{1} & =-(2-\alpha) \int_{|z|<\delta}\left(\left(D^{2} \phi\left(y_{\alpha}\right)+o_{\delta}(1)\right) z, z\right) d \mu_{\alpha} \\
& =-(2-\alpha) \int_{|z|<\delta}\left(D^{2} \phi\left(y_{\alpha}\right) z, z\right) d \mu_{\alpha}+o_{\delta}(1) .
\end{aligned}
$$

Note that the $o_{\delta}(1)$-term is independent of $\alpha$ because the measure $(2-\alpha)|z|^{2} \mu_{\alpha}$ has bounded mass. The symmetry of $\mu_{\alpha}$ implies that $\int_{|z|<\delta} z_{i} z_{j} d \mu_{\alpha}=0$ and then, by Lemma 7.2, we get

$$
I_{1}=-(2-\alpha) \operatorname{Tr}\left(D^{2} \phi\left(y_{\alpha}\right)\right) \int_{|z|<\delta}|z|^{2} d \mu_{\alpha}+o_{\delta}(1)=a \Delta \phi(x)+o_{\alpha}(1)+o_{\delta}(1) .
$$

Similarly we have

$$
I_{2}=-(2-\alpha) \int_{|z|<\delta} D \phi\left(y_{\alpha}\right) \cdot z d \mu_{\alpha}=-(2-\alpha) D \phi\left(y_{\alpha}\right) \int_{|z|<\delta} z d \mu_{\alpha},
$$

and by symmetry of $\mu_{\alpha}$ and Lemma 7.2 we see that

$$
I_{2}=-(2-\alpha) D \phi\left(y_{\alpha}\right) \int_{|z|<\delta} z \frac{g(z)-g(0)}{|z|^{N+\alpha}} d z=b \cdot D \phi(x)+o_{\alpha}(1)+o_{\delta}(1) .
$$

The $o_{\delta}(1)$-terms are independent of $\alpha$ since the measures $\nu_{\alpha}^{2}$ of Lemma 7.2 have unformly bounded mass. For the last integral $I_{3}$, we use the boundedness of $\left(u_{\alpha}\right)_{\alpha}$ with respect to $\alpha$ to see that

$$
\left|I_{3}\right| \leq C(2-\alpha) \int_{|z| \geq \delta} \frac{d z}{|z|^{N+\alpha}} \leq C^{\prime} \frac{2-\alpha}{\alpha \delta^{\alpha}} \rightarrow 0 \quad \text { as } \quad \alpha \rightarrow 2 .
$$

So we keep $\delta>0$ fixed and pass to the limit as $\alpha \rightarrow 2^{-}$(and $y_{\alpha} \rightarrow x$ ) to get

$$
-a \Delta \phi(x)-b \cdot D \phi(x)+\bar{u}(x) \leq f(x)+o_{\delta}(1) .
$$

Then, since $\delta<x_{N}$ could be arbitrarily small, we pass to the limit as $\delta \rightarrow 0$ and get the viscosity subsolution condition for $\bar{u}$ at $x$.

Step 2. Case (ii) where $x \in \partial \Omega$, that is, $x_{N}=0$. We again consider a smooth function $\phi$ such that $\bar{u}-\phi$ has a strict local maximum point at $x$ and, as above, we have a sequence $\left(y_{\alpha}\right)_{\alpha}$ of maximum points of $u_{\alpha}-\phi$ such that $y_{\alpha} \rightarrow x$ as $\alpha \rightarrow 2^{-}$.

In this step we are going to prove that

$$
\min \left(-\mathcal{M}^{+}\left(D^{2} u(x)\right)-\Lambda|D u(x)|+\bar{u}(x)-f(x) ; \frac{\partial \phi}{\partial \mathbf{n}}(x)\right) \leq 0,
$$

where $\mathcal{M}^{+}$is defined in (7.3). We may assume that $\frac{\partial \phi}{\partial \mathbf{n}}(x)=-\frac{\partial \phi}{\partial x_{N}}(x)>0$ since otherwise (7.5) is already satisfied. Then for $\alpha$ close to $2,-\frac{\partial \phi}{\partial x_{N}}\left(y_{\alpha}\right)>0$ by the 
continuity of $D \phi$. We can also assume $y_{\alpha} \in \Omega$, since otherwise $y_{\alpha} \in \partial \Omega$ and then $\frac{\partial \phi}{\partial \mathbf{n}}\left(y_{\alpha}\right)=-\frac{\partial \phi}{\partial x_{N}}\left(y_{\alpha}\right) \leq 0$ for $\alpha$ close to 2 by Definition 2.3. and this would contradict our assumption.

Therefore $0<y_{\alpha, N} \rightarrow 0$ as $\alpha \rightarrow 2$, and the subsolution inequality for $u_{\alpha}$ takes the form

$$
\begin{aligned}
& -(2-\alpha) \int_{|z|<\delta} \phi\left(y_{\alpha}+\eta\left(y_{\alpha}, z\right)\right)-\phi\left(y_{\alpha}\right)-D \phi\left(y_{\alpha}\right) \cdot \eta\left(y_{\alpha}, z\right) d \mu_{\alpha} \\
& -(2-\alpha) \int_{|z|<\delta} D \phi\left(y_{\alpha}\right) \cdot \eta\left(y_{\alpha}, z\right) d \mu_{\alpha} \\
& -(2-\alpha) \int_{|z| \geq \delta} u_{\alpha}\left(P\left(y_{\alpha}\right)-u_{\alpha}\left(y_{\alpha}\right) d \mu_{\alpha}+u_{\alpha}\left(y_{\alpha}\right) \leq f\left(y_{\alpha}\right) .\right.
\end{aligned}
$$

We denote as before the three integral terms by $I_{1}, I_{2}, I_{3}$. The compensator term $I_{2}$ can be written as

$$
\begin{aligned}
I_{2}= & -g(0) D \phi\left(y_{\alpha}\right) \cdot(2-\alpha) \int_{|z|<\delta} \eta\left(y_{\alpha}, z\right) \frac{d z}{|z|^{N+\alpha}} \\
& -D \phi\left(y_{\alpha}\right) \cdot(2-\alpha) \int_{|z|<\delta} \eta\left(y_{\alpha}, z\right) \frac{g(z)-g(0)}{|z|^{N+\alpha}} d z \\
= & I_{2,1}+I_{2,2} .
\end{aligned}
$$

For symmetry reasons of both $\eta$ and the measure, $I_{2,1}$ reduces to the scalar product of the $N$-th components, and it has a sign,

$$
I_{2,1}=-g(0) \frac{\partial \phi}{\partial x_{N}}\left(y_{\alpha}\right)(2-\alpha) \int_{|z|<\delta} \eta\left(y_{\alpha}, z\right)_{N} \frac{d z}{|z|^{N+\alpha}} \geq 0,
$$

since $g(0),-\frac{\partial \phi}{\partial x_{N}}\left(y_{\alpha}\right)$, and the $\eta$-integral are nonnegative (see Lemma 2.8 (iii)). Thus we may drop the $I_{2,1}$-term from the inequality above and get that

$$
I_{1}+I_{2,2}+I_{3}+u_{\alpha}\left(y_{\alpha}\right) \leq f\left(y_{\alpha}\right) .
$$

We now pass to the limit in this inequality as $\alpha \rightarrow 2$, and hence $y_{\alpha, N} \rightarrow 0$. The difference with Step 1 above is that now $y_{\alpha}$ converges to the boundary so that we cannot take a fixed $0<\delta<y_{\alpha, N}$ as $\alpha \rightarrow 2$. For the first integral, Lemma 7.2 enables us to take subsequences $\alpha_{k} \rightarrow 2$ and $y_{\alpha} \rightarrow 0$ such that (dropping the subscript $k$ for simplicity)

$$
\begin{aligned}
I_{1} & =-(2-\alpha) \int_{|z|<\delta} \phi\left(y_{\alpha}+\eta\left(y_{\alpha}, z\right)\right)-\phi\left(y_{\alpha}\right)-D \phi\left(y_{\alpha}\right) \cdot \eta\left(y_{\alpha}, z\right) \frac{g(z) d z}{|z|^{N+\alpha}} \\
& =-\sum_{i, j} \int_{|z|<\delta}\left(\partial_{i, j}^{2} \phi\left(y_{\alpha}\right)+o_{\delta}(1)\right) d\left(\nu_{\alpha, y_{\alpha}}^{3}\right)_{i, j}(z) \\
& =-\sum_{i, j} \partial_{i, j}^{2} \phi(x) \int_{|z|<\delta} d\left(\nu_{\alpha, y_{\alpha}}^{3}\right)_{i, j}(z)+o_{\alpha}(1)+o_{\delta}(1) \\
& =-\sum_{i} \bar{a}_{i}(x) \partial_{i, i}^{2} \phi(x)+o_{\alpha}(1)+o_{\delta}(1) \\
& \geq-\mathcal{M}^{+}\left(D^{2} \phi(x)\right)+o_{\alpha}(1)+o_{\delta}(1) .
\end{aligned}
$$

The last term $I_{3}$ can be treated as in Step 1 and vanishes as $\alpha \rightarrow 2$. We are left with the $I_{2,2}$-term and again use Lemma 7.2, this time for the measure $\nu^{4}$. The 
result is the existence of a vector $\bar{b}(x)$ such that along subsequences we have

$$
\begin{aligned}
I_{2,2} & =D \phi\left(y_{\alpha}\right) \cdot(2-\alpha) \int_{|z|<\delta} \eta\left(y_{\alpha}, z\right) \frac{g(z)-g(0)}{|z|^{N+\alpha}} d z \\
& =D \phi(x) \cdot \bar{b}(x)+o_{\alpha}(1) \geq-\Lambda|D \phi(x)|+o_{\alpha}(1) .
\end{aligned}
$$

Hence, passing to the limit $\alpha \rightarrow 2$ in the above inequality leads to

$$
-\mathcal{M}^{+}\left(D^{2} \phi(x)\right)-\Lambda|D \phi(x)|+\bar{u}(x)-f(x) \leq 0,
$$

and (7.5) still holds.

Step 3. We shall now prove that boundary condition (7.5) reduces to the condition $\frac{\partial \phi}{\partial \mathbf{n}} \leq 0$. Let us assume on the contrary that $\frac{\partial \phi}{\partial \mathbf{n}}(x)>0$ for some point $x$ at the boundary $\left\{x_{N}=0\right\}$ and some smooth function $\phi$ such that $u-\phi$ has a maximum point at $x$. For any $\tau, \varepsilon>0$, we take a smooth, bounded function $\psi: \mathbb{R}_{+} \rightarrow \mathbb{R}_{+}$ such that

$$
\psi(t)=\tau\left(t-\frac{t^{2}}{\varepsilon^{2}}\right) \quad \text { for } \quad 0 \leq t \leq \varepsilon^{2} / 2 .
$$

Since $\psi(0)=0$ and $0 \leq \psi$ for $0 \leq t \leq \varepsilon^{2} / 2$, it follows that $u(x)-\phi(x)-\psi\left(x_{N}\right)$ again has a local maximum point at $x$. Hence (7.5) holds with $\phi(x)+\psi\left(x_{N}\right)$ replacing $\phi(x)$, i.e.

$$
\min \left(E(\phi)+\frac{a}{2} \frac{2 \tau}{\varepsilon^{2}}-\Lambda \tau ; \frac{\partial \phi}{\partial \mathbf{n}}(x)-\tau\right) \leq 0
$$

where

$$
E(\phi):=-\mathcal{M}^{+}\left(D^{2} \phi(x)\right)-\Lambda|D \phi(x)|+\bar{u}(x)-f(x) .
$$

Since we assumed that $\frac{\partial \phi}{\partial \mathbf{n}}(x)>0$, we first fix $\tau>0$ small enough so that the inequality $\frac{\partial \phi}{\partial \mathbf{n}}(x)-\tau>0$ still holds. Then we can choose $\varepsilon>0$ small enough to ensure that also

$$
E(\phi)+\frac{a}{2} \frac{2 \tau}{\varepsilon^{2}}-\Lambda \tau>0 .
$$

But then we contradict (7.6), and hence the boundary condition for $\bar{u}$ reduces to $\partial \phi / \partial \mathbf{n} \leq 0$ everywhere on the boundary. This concludes the proof of Proposition 7.4

Proof of Theorem 7.1. We have seen that $\bar{u}$ is a subsolution of (7.2) while $\underline{u}$ is a supersolution of the same problem. Since $\underline{u} \leq \bar{u}$ on $\bar{\Omega}$ by definition and $\underline{u} \geq \bar{u}$ on $\bar{\Omega}$ by the comparison principle for (7.2), we see that $\underline{u}=\bar{u}$ on $\bar{\Omega}$. Setting $u:=\underline{u}=\bar{u}$ on $\bar{\Omega}$, it immediately follows that $u$ is continuous (since $\underline{u}$ is lsc and $\bar{u}$ is usc) and the unique viscosity solution of (7.2). By classical arguments in the half-relaxed limit method, the sequence $\left(u_{\alpha}\right)_{\alpha}$ also converges locally uniformly to $u$.

\section{Appendix A. Blow-up supersolution in Censored CASE. I}

In this section we assume $\left(\mathrm{H}_{\eta}^{6}\right)$ and $\left(\mathrm{H}_{\mu}^{\prime}\right)$ as in Section 5. Remeber that $\Omega:=$ $\left\{\left(x_{1}, \ldots, x_{N}\right)=\left(x^{\prime}, x_{N}\right): x_{N} \geq 0\right\}$. First we show that in the censored fractional Laplace case (i.e. the censored alpha stable case), we can essentially take

$$
\mathcal{U}(x)=-\ln x_{N}
$$

as our blow-up supersolution in assumption (U) in Section 5. 
Lemma A.1. If $d \mu(z)=\frac{d z}{|z|^{N+\alpha}}$ for $\alpha \in(0,1)$ and $\mathcal{U}(x)=-\ln \left(x_{N}\right)$, then

$$
-I[\mathcal{U}](x)=-\int_{x_{N}+z_{N} \geq 0} \mathcal{U}(x+z)-\mathcal{U}(x) \frac{d z}{|z|^{N+\alpha}}>0 \quad \text { for } \quad x \in \Omega .
$$

Proof. We first change variables, $\bar{z}=\frac{z}{x_{N}}$, to find that

$$
-I[\mathcal{U}](x)=\int_{x_{N}+z_{N} \geq 0} \ln \left(1+\frac{z_{N}}{x_{N}}\right) \frac{d z}{|z|^{N+\alpha}}=\frac{1}{x_{N}^{\alpha}} \int_{\bar{z}_{N} \geq-1} \ln \left(1+\bar{z}_{N}\right) \frac{d \bar{z}}{|\bar{z}|^{N+\alpha}} .
$$

Now we are done if we can prove that

$$
J=\int_{\bar{z}_{N} \geq-1} \ln \left(1+\bar{z}_{N}\right) \frac{d \bar{z}}{|\bar{z}|^{N+\alpha}}>0 .
$$

When $N=1$, we take $1+\bar{z}=e^{y}$ and note that simple computations lead to

$$
J=\int_{-\infty}^{\infty} y \frac{e^{y} d y}{\left|e^{y}-1\right|^{1+\alpha}}=\int_{-\infty}^{\infty} F(y) e^{\frac{y}{2}(1-\alpha)} d y \quad \text { where } \quad F(y)=\frac{y}{\left|2 \sinh \frac{y}{2}\right|^{1+\alpha}} .
$$

Since $F(y)$ is odd and $1-\alpha>0$,

$$
0<-F(-y) e^{-\frac{y}{2}(1-\alpha)}<F(y) e^{\frac{y}{2}(1-\alpha)} \quad \text { for } \quad y>0,
$$

and hence by symmetry $J>0$.

In the case $N>1$ we introduce polar coordinates $z=r y$ where $r \geq 0$ and $|y|=1$, and we let $d S(y)$ be the surface measure on the sphere $|y|=1$ in $\mathbb{R}^{N}$. We then find that

$$
J=\left(\int_{|y|=1, y_{N}>0} \int_{0}^{\infty}+\int_{|y|=1, y_{N}<0} \int_{0}^{-\frac{1}{y_{N}}}\right) \ln \left(1+r y_{N}\right) \frac{r^{N-1} d r d S(y)}{r^{N+\alpha}} .
$$

The change of variables $s=y_{N} r$ then leads to

$$
\begin{aligned}
J & =\left(\int_{|y|=1, y_{N}>0} \int_{0}^{\infty}+\int_{|y|=1, y_{N}<0} \int_{0}^{-1}\right) \operatorname{sgn}\left(y_{N}\right)\left|y_{N}\right|^{\alpha} \ln (1+s) \frac{d s}{|s|^{1+\alpha}} d S(y) \\
& =\int_{|y|=1, y_{N}>0}\left|y_{N}\right|^{\alpha} d S(y) \int_{-1}^{\infty} \ln (1+s) \frac{d s}{|s|^{1+\alpha}} .
\end{aligned}
$$

The lemma now follows from the computations we did for $N=1$.

We now generalize to a much larger class of integral operators with Lévy measures $\mu$ such that $d \mu(z) \sim \frac{d z}{|z|^{N+\alpha}}$ near $|z|=0$. In this case the blow-up supersolution will be the modified log-function $\mathcal{U}_{R}$ defined as

$$
\mathcal{U}_{R}(x)=\overline{\mathcal{U}}_{R}\left(x_{N}\right) \quad \text { for } \quad x \in \Omega, \quad R>1,
$$

where $\overline{\mathcal{U}}_{R}$ is a (nonnegative) monotone decreasing $C^{\infty}(0, \infty)$ function such that

$$
\overline{\mathcal{U}}_{R}(s)= \begin{cases}-\ln (s)+\frac{3}{2} \ln R & \text { if } 0<s \leq R \\ 0 & \text { if } s \geq 2 R\end{cases}
$$


The main result in this appendix says that $\mathcal{U}_{R}$ will be the blow-up "supersolution" of assumption (U) provided the Lévy measure $\mu$ also satisfies

$(U)^{\prime}$ For all $R, \varepsilon>0$ there are $r, c, K>0$ and $\alpha \in(0,1)$ such that
(a) $\int_{-1<z_{N} \leq R} \ln \left(1+z_{N}\right)\left(s^{\alpha} \mu(s d z)-\frac{c d z}{|z|^{N+\alpha}}\right)>-\varepsilon \quad$ for $\quad s \in(0, r)$,
(b) $\int_{-1<z_{N} \leq-\frac{1}{2}} \ln \left(1+z_{N}\right) \mu(s d z) \geq-K$
for $s \in(r, R)$.

Theorem A.2. Assume $\left(\mathrm{H}_{\eta}^{6}\right)$, $\left(\mathrm{H}_{\mu}\right)^{\prime}$, and $(U)^{\prime}$ hold. Then the function $\mathcal{U}_{R}$ defined above satisfies the assumptions in $(\mathrm{U})$. In particular, there is $R_{0}>0$ such that for any $R>R_{0}$ there is $K_{R} \geq 0$ such that

$$
-I\left[\mathcal{U}_{R}\right](x) \geq-K_{R} \quad \text { in } \quad\left\{x: 0<x_{N} \leq R\right\} .
$$

Before we prove this result, we show how assumption $(U)^{\prime}$ can be checked when $\mu$ is a Lévy measure whose restriction to $\left\{z:\left|z_{N}\right| \leq r\right\}$ has a density

$$
\frac{d \mu}{d z}=\frac{g(z)}{|z|^{N+\alpha}}, \quad \text { where } \quad\left\{\begin{array}{l}
\alpha \in(0,1) \\
0 \leq g \in L_{\mathrm{loc}}^{\infty}\left(\mathbb{R}^{N}\right) \cap L^{1}\left(\mathbb{R}^{N} ; \frac{d z}{1+|z|^{N+\alpha}}\right) \\
\lim _{z \rightarrow 0} g(z)=g(0)>0
\end{array}\right.
$$

Note that the $L^{1}$ assumption makes $\frac{d \mu}{d z}$ integrable near infinity and that $L^{\infty}\left(\mathbb{R}^{N}\right) \subset$ $L^{1}\left(\mathbb{R}^{N} ; \frac{d z}{1+|z|^{N+\alpha}}\right)$ for $\alpha>0$.

Corollary A.3. If $\mu$ has a density satisfying (A.1), then the function $\mathcal{U}_{R}$ defined above satisfies the assumptions in $(\mathrm{U})$.

Proof. By Theorem A.2 the proof consists in checking that $(U)^{\prime}$ holds. Part (b) follows from Hölder's inequality since $\ln (1+s) \in L^{1}(-1,0)$. Now we check part (a). Note that

$$
s^{\alpha} \mu(s d z)-\frac{c d z}{|z|^{N+\alpha}}=\frac{g(s z)-c}{|z|^{N+\alpha}} d z .
$$

Now choose $c=g(0)$ and write

$$
\begin{aligned}
& \int_{-1<z_{N} \leq R} \ln \left(1+z_{N}\right)\left(s^{\alpha} \mu(s d z)-\frac{c d z}{|z|^{N+\alpha}}\right) \\
& \geq-\sup _{-s<r<R s}|g(r)-g(0)| \int_{-1<z_{N} \leq R}\left|\ln \left(1+z_{N}\right)\right| \frac{d z}{|z|^{N+\alpha}} .
\end{aligned}
$$

Part (a) now follows since the last integral is finite for any $R>0$, while the sup-term goes to zero as $s \rightarrow 0$ by continuity of $g$ at $z=0$.

Remark A.4. Assumption (A.1) also includes measures like

$$
\mu=\sum_{i=1}^{M_{1}} \mu_{i},
$$

where each measure $\mu_{i}$ has a density satisfying (A.1) for different $g_{i}$ and $\alpha_{i}$. To see this, simply take $\alpha=\max _{i} \alpha_{i}$ and $g(z)=\sum_{i=1}^{M} g_{i}(z)|z|^{\alpha-\alpha_{i}}$ and note that $g \in L^{1}\left(\mathbb{R}^{N} ; \frac{d z}{1+|z|^{N+\alpha}}\right)$. We can even relax this assumption to include measures with zero or arbitrary negative $\alpha_{i}$ provided that $\max _{i} \alpha_{i}$ remains in $(0,1)$. Finally we 
mention that we need some assumption on $\mu$ in order to control the negative part of the integral $-I\left[\mathcal{U}_{R}\right]$. In A.1 we do this by requiring continuity at 0 of $g$, but a careful reader can extend this assumption to allow some discontinuities at 0 .

Remark A.5. In assumption $(U)^{\prime}$ it is only the restriction of $\mu$ to the set

$$
\left\{z:-r<z_{N}<R r\right\} \cap\left\{z:-1<z_{N}<-\frac{1}{2}\right\}
$$

that plays any role. Hence if $\mu$ satisfies $(U)^{\prime}$, by taking $\bar{r}$ small enough, so will $\mu+\bar{\mu}$ for any measure $\bar{\mu}$ satisfying

$$
\int_{|z|>0} d \bar{\mu}<\infty \quad \text { and } \quad \operatorname{supp} \bar{\mu} \cap\left\{z:-1<z_{N}<\bar{r}\right\}=\emptyset \quad \text { for some } \bar{r}>0 .
$$

For example, the delta-measure $\bar{\mu}=\sum_{i=1}^{M} \delta_{x^{i}}$ is ok if $x_{N}^{i}>0$.

Proof of Theorem A.2. First note that there is an $R_{0}>0$ such that

$$
J_{R_{0}}:=\int_{-1<z_{N} \leq R_{0}} \ln \left(1+z_{N}\right) \frac{d z}{|z|^{N+\alpha}}>0 .
$$

Indeed, in the proof of Lemma A.1, we showed that $J_{\infty}=J>0$. The result then follows by the Dominated Convergence Theorem since the integrand is positive for $z_{N}>0$ and integrable.

For any $R>R_{0}$, we note immediatly that $\mathcal{U}_{R}$ is a nonnegative decreasing function which trivially satisfies the second part of $(\mathrm{U})$ with $\omega_{R}(s)=\frac{1}{\mathcal{U}_{R}(s)}$. We will now check that $\mathcal{U}_{R}$ has the appropriate supersolution properties and hence complete the proof that $\mathcal{U}_{R}$ satisfies $(\mathrm{U})$ under $(U)^{\prime}$. By the definition of $\mathcal{U}_{R}$, we can write

$$
\begin{aligned}
-I\left[\mathcal{U}_{R}\right](x) & =\int_{-x_{N}<z_{N} \leq R x_{N}} \ln \left(1+\frac{z_{N}}{x_{N}}\right) \mu(d z)+I_{R} \\
& =\int_{-1<y_{N} \leq R} \ln \left(1+y_{N}\right) \mu\left(x_{N} d y\right)+I_{R},
\end{aligned}
$$

where $I_{R}=-\int_{z_{N}>R x_{N}} \mathcal{U}_{R}(x+z)-\mathcal{U}_{R}(x) \mu(d z)>0$ since $\mathcal{U}_{R}$ is decreasing. By assumption $(U)^{\prime}$ we then find an $r>0$ such that for $x_{N} \in(0, r)$,

$$
-x_{N}^{\alpha} I\left[\mathcal{U}_{R}\right](x) \geq J_{R}+\int_{-1<y_{N} \leq R} \ln (1+y)\left(x_{N}^{\alpha} \mu\left(x_{N} d y\right)-\frac{d y}{|y|^{1+\alpha}}\right) \geq \frac{1}{2} J_{R}>0 .
$$

When $x_{N} \in(r, R)$, another application of $(U)^{\prime}$ along with $\left(\mathrm{H}_{\mu}\right)^{\prime}$ leads to

$$
\begin{aligned}
& -I\left[\mathcal{U}_{R}\right](x) \\
& \geq\left(\int_{-x_{N}<z_{N}<-\frac{x_{N}}{2}}+\int_{-\frac{x_{N}}{2}<z_{N}<R \cap|z|<1}+\int_{-\frac{x_{N}}{2}<z_{N}<R \cap|z|>1}\right) \ln \left(1+\frac{z}{x_{N}}\right) d \mu(d z) \\
& \geq-K-\max _{s \in\left(-\frac{1}{2}, \frac{R}{r}\right)} \frac{\left|\partial_{s} \ln (1+s)\right|}{\left|x_{N}\right|} \int_{|z|<1}|z| d \mu(z)-\max _{s \in\left(-\frac{1}{2}, \frac{R}{r}\right)}|\ln (1+s)| \int_{|z|>1} d \mu(z) .
\end{aligned}
$$

Since this last expression is bounded for $x_{N} \in(r, R)$, this completes the proof. 
Appendix B. Estimates for the CEnsored CASE. II

Lemma B.1. Let $\mu(d z)=\frac{d z}{|z|^{N+\alpha}}, \alpha \in(1,2)$, and define $\tilde{\theta}(x)=\left|x_{N}\right|^{\beta}$. If $\beta \in(0,1)$ and $x \in \Omega$, then

$$
I[\tilde{\theta}](x)=\mathrm{P} . \mathrm{V} \cdot \int_{x_{N}+z_{N} \geq 0} \tilde{\theta}(x+z)-\tilde{\theta}(x) \mu(d z) \begin{cases}>0 & \text { if } \beta>\alpha-1 \\ =0 & \text { if } \beta=\alpha-1 \\ <0 & \text { if } \beta<\alpha-1\end{cases}
$$

One of the referees informed us that this result is known and a proof can be found in [9]. Our proof is different and we keep it for the reader's convenience.

Proof. First let $\beta \in(0,1)$ and $N=1$, and define $\tilde{\theta}(x)=|x|^{\beta}$. Note that the change of variables $z=x \bar{z}$ followed by $1+\bar{z}=e^{s}$ reveals that

$$
\begin{aligned}
I[\tilde{\theta}](x) & =\mathrm{P} . \mathrm{V} \cdot \int_{x+z \geq 0}|x+z|^{\beta}-|x|^{\beta} \frac{d z}{|z|^{1+\alpha}} \\
& =|x|^{\beta-\alpha} \mathrm{P} . \mathrm{V} \cdot \int_{\bar{z} \geq-1}|1+\bar{z}|^{\beta}-1 \frac{d \bar{z}}{|\bar{z}|^{1+\alpha}} \\
& =|x|^{\beta-\alpha} \text { P.V. } \int_{-\infty}^{\infty} \frac{2 \sinh \frac{\beta s}{2}}{\left|2 \sinh \frac{s}{2}\right|^{1+\alpha}} e^{\frac{s}{2}(1+\beta-\alpha)} d x .
\end{aligned}
$$

When $\beta=\alpha-1$, the integrand is odd and hence the integral is zero. For $\beta>\alpha-1$ $(\beta<\alpha-1)$ the exponential factor makes the integral positive (negative). Hence when $\beta+1-\alpha=0,>0$ or $<0$, then $I[\tilde{\theta}]=0,>0$, or $<0$ respectively.

When $N>1$, a similar result holds for $\tilde{\theta}(x)=\left|x_{N}\right|^{\beta}$. The idea is to work in polar coordinates. We set $x=r y$ for $r \geq 0$ and $|y|=1$ and let $d S(y)$ denote the surface area element of the $N$-sphere $|y|=1$. We also use the change of variables $r y_{N}=\bar{r} x_{N}$.

$$
\begin{aligned}
& I[\tilde{\theta}](x) \\
& =\int_{x_{N}+z_{N} \geq 0}\left|x_{N}+z_{N}\right|^{\beta}-\left|x_{N}\right|^{\beta} \frac{d z}{|z|^{N+\alpha}} \\
& =\int_{|y|=1} \int_{x_{N}+r y_{N}>0}\left|x_{N}+r y_{N}\right|^{\beta}-\left|x_{N}\right|^{\beta} \frac{r^{N-1} d r d S(y)}{r^{N+\alpha}} \\
& =\left(\int_{|y|=1, y_{N}>0} \int_{0}^{\infty}+\int_{|y|=1, y_{N}<0} \int_{0}^{-\frac{x_{N}}{y_{N}}}\right)(\cdots) \frac{d r d S(y)}{r^{1+\alpha}} \\
& =\left(\int_{|y|=1, y_{N}>0} \int_{0}^{\infty}-\int_{|y|=1, y_{N}<0} \int_{0}^{-1}\right)\left|x_{N}\right|^{\beta-\alpha}\left|y_{N}\right|^{\alpha}\left(|1+\bar{r}|^{\beta}-1\right) \frac{d \bar{r} d S(y)}{|\bar{r}|^{1+\alpha}} \\
& =\left|x_{N}\right|^{\beta-\alpha} \int_{|y|=1, y_{N}>0}\left|y_{N}\right|^{\alpha} d S(y) \int_{-1}^{\infty 1+\left.\bar{r}\right|^{\beta}-1 \frac{d \bar{r}}{|\bar{r}|^{1+\alpha}} .}
\end{aligned}
$$

Here the first integral is just a positive constant while the second integral is the same as the one we found in the $N=1$ case. The conclusion is therefore as in that case: When $\beta+1-\alpha=0,>0$ or $<0$, then $I[\tilde{\theta}]=0,>0$, or $<0$ respectively. 
Next we consider the two integrals

$$
\begin{aligned}
B(a) & =\int_{-a-1}^{-a}|1+z|^{\beta}-1 d \mu(z), \\
G & =\int_{-1}^{1}|1+z|^{\beta}+|1-z|^{\beta}-2 d \mu(z),
\end{aligned}
$$

where $a>1, \beta \in(0,1)$, and $d \mu(z)=\frac{d z}{|z|^{N+\alpha}}$ for $\alpha \in[1,2)$.

Proposition B.2. If $\beta=\alpha-1$, then there is $\kappa>0$ such that

$$
B(a)+G \leq-\kappa<0
$$

for any $a>1$.

By continuity of the integrals in $\beta$ we have the following corollary:

Corollary B.3. There is $\kappa>0$ and $\beta>\alpha-1$ such that

$$
B(a)+G \leq-\kappa \leq 0
$$

for any $a>1$.

To prove Proposition B.2, note that $z+1 \leq 0$ for $z \in(-a-1,-a)(a>1)$ and that the change of variable $1+z=-e^{x}$ in $B(a)$ leads to

$$
B(a)=\int_{\ln (a-1)}^{\ln a} \frac{2 \sinh \frac{\beta x}{2}}{\left|2 \cosh \frac{x}{2}\right|^{1+\alpha}} e^{\frac{x}{2}(1+\beta-\alpha)} d x \stackrel{\beta=\alpha-1}{=} \int_{\ln (a-1)}^{\ln a} \frac{2 \sinh \frac{\beta x}{2}}{\left|2 \cosh \frac{x}{2}\right|^{1+\alpha}} d x .
$$

For the $G$ integral we have the following result.

\section{Lemma B.4.}

$G=2 \mathrm{P} . \mathrm{V} . \int_{-\ln 2}^{\ln 2} \frac{2 \sinh \frac{\beta x}{2}}{\left|2 \sinh \frac{x}{2}\right|^{1+\alpha}} e^{\frac{x}{2}(1+\beta-\alpha)} d x-2 \int_{\ln 2}^{\infty} \frac{2 \sinh \frac{\beta x}{2}}{\left|2 \sinh \frac{x}{2}\right|^{1+\alpha}} e^{-\frac{x}{2}(1+\beta-\alpha)} d x$, and if $\beta=\alpha-1$,

$$
G=-2 \int_{\ln 2}^{\infty} \frac{2 \sinh \frac{\beta x}{2}}{\left|2 \sinh \frac{x}{2}\right|^{1+\alpha}} d x
$$

Proof. First note that by symmetry

$$
G=2 \lim _{b \rightarrow 0^{+}} \int_{(-1,1) \backslash(-b, b)}|1+z|^{\beta}-1 d \mu(z) .
$$

Then, since $1+z>0$ for $z \in(-1,1)$, the change of variable $1+z=e^{x}$ leads to

$$
G=2 \lim _{b \rightarrow 0^{+}} \int_{(-\infty, \ln 2) \backslash(\ln (1-b), \ln (1+b))} \frac{2 \sinh \frac{\beta x}{2}}{\left|2 \sinh \frac{x}{2}\right|^{1+\alpha}} e^{\frac{x}{2}(1+\beta-\alpha)} d x .
$$

Note that $\ln (1 \pm b)= \pm b+O\left(b^{2}\right)$ and decompose the above integral as follows:

$$
\begin{aligned}
& \int_{(-\infty, \ln 2) \backslash(\ln (1-b), \ln (1+b))}(\cdots) d x \\
& =\left(\int_{(-\infty, \ln 2) \backslash(-b,+b)}+\int_{(-\infty, \ln 2) \backslash(\ln (1-b),-b)}-\int_{(-\infty, \ln 2) \backslash(\ln (1+b), b)}\right)(\cdots) d x .
\end{aligned}
$$


Now since $\sinh x=x+O\left(x^{3}\right)$, the last two integrals are bounded by $C b^{2} \frac{b}{b^{1+\alpha}}=$ $C b^{2-\alpha}$ for $b \ll 1$, and we have

$$
G=2 \lim _{b \rightarrow 0^{+}} \int_{(-\infty, \ln 2) \backslash(-b, b)}(\cdots) d x=2\left(\text { P.V. } \int_{(-\ln 2, \ln 2)}+\int_{(-\infty,-\ln 2)}\right)(\cdots) d x .
$$

A change of variables in the last integral then gives the first statement of the lemma. The last part of the lemma follows since the integrand is odd when $\beta=\alpha-1$, and hence the integral over $(-\ln 2, \ln 2)$ vanishes.

We also need the next lemma.

Lemma B.5. If $\beta=\alpha-1$, then $B(2)<-\frac{G}{2}$.

Proof. We will show that

$$
B(2)=\int_{0}^{\ln 2} \frac{2 \sinh \frac{\beta x}{2}}{\left|2 \cosh \frac{x}{2}\right|^{1+\alpha}} d x \leq \int_{0}^{\ln 2} \frac{2 \sinh \frac{\beta(x+\ln 2)}{2}}{\left|2 \sinh \frac{x+\ln 2}{2}\right|^{1+\alpha}} d x<\int_{0}^{\infty}(\cdots) d x=-\frac{G}{2} .
$$

The last inequality is trivial, and since sinh is an increasing function, the first inequality follows if we can show that

$$
\cosh \frac{x}{2} \geq \sinh \frac{x+\ln 2}{2} \text { for all } x \in(0, \ln 2) .
$$

But this easily follows since $f(x)=\cosh \frac{x}{2}-\sinh \frac{x+\ln 2}{2}$ satisfies

$$
\begin{aligned}
f^{\prime}(x) & =\frac{1}{2} \sinh \frac{x}{2}-\frac{1}{2} \cosh \frac{x+\ln 2}{2} \leq 0 \quad \text { for all } x, \\
f(\ln 2) & =\frac{\sqrt{2}-1}{4} \geq 0 .
\end{aligned}
$$

Proof of Proposition B.2, Divide the integral $B(a)$ into three parts

$$
\left(\int_{\ln (a-1) \wedge 0}^{0}+\int_{\ln (a-1) \vee 0}^{\ln 2 \wedge \ln a}+\int_{\ln 2}^{\ln 2 \vee \ln a}\right)(\cdots) d x .
$$

Now we conclude since the first integral is negative, the second one is less than $-\frac{G}{2}$ by Lemma B.5, and the last one is less than $-\frac{G}{2}$ by definition of $G$.

\section{ACKNOWLEDGMENTS}

The authors wish to warmly thank the anonymous referee for raising some important questions that allowed them to improve some proofs as well as the presentation of the original manuscript.

\section{REFERENCES}

[1] David Applebaum, Lévy processes and stochastic calculus, 2nd ed., Cambridge Studies in Advanced Mathematics, vol. 116, Cambridge University Press, Cambridge, 2009. MR 2512800 (2010m:60002)

[2] Martino Bardi and Italo Capuzzo-Dolcetta, Optimal control and viscosity solutions of Hamilton-Jacobi-Bellman equations, Systems \& Control: Foundations \& Applications, Birkhäuser Boston Inc., Boston, MA, 1997. With appendices by Maurizio Falcone and Pierpaolo Soravia. MR 1484411 (99e:49001) 
[3] Guy Barles, Solutions de viscosité des équations de Hamilton-Jacobi (French, with French summary), Mathématiques \& Applications (Berlin) [Mathematics \& Applications], vol. 17, Springer-Verlag, Paris, 1994. MR.1613876 (2000b:49054)

[4] G. Barles, E. Chasseigne, and C. Imbert, On the Dirichlet problem for second-order elliptic integro-differential equations, Indiana Univ. Math. J. 57 (2008), no. 1, 213-246, DOI 10.1512/iumj.2008.57.3315. MR2400256(2009b:35088)

[5] G. Barles, C. Georgelin, and E. Jakobsen. On Neumann and oblique derivatives boundary conditions for nonlocal elliptic equations, Journal of Differential Equations 256 (2014), 1368 1394. MR3145761

[6] Guy Barles and Cyril Imbert, Second-order elliptic integro-differential equations: viscosity solutions' theory revisited, Ann. Inst. H. Poincaré Anal. Non Linéaire 25 (2008), no. 3, 567585, DOI 10.1016/j.anihpc.2007.02.007. MR2422079 (2009c:35102)

[7] Guy Barles and Pierre-Louis Lions, Remarques sur les problèmes de réflexion oblique (French, with English and French summaries), C. R. Acad. Sci. Paris Sér. I Math. 320 (1995), no. 1, 69-74. MR 1320834 (95k:60137)

[8] Richard F. Bass and Moritz Kassmann, Hölder continuity of harmonic functions with respect to operators of variable order, Comm. Partial Differential Equations 30 (2005), no. 7-9, 12491259, DOI 10.1080/03605300500257677. MR2180302(2006i:31005)

[9] Krzysztof Bogdan, Krzysztof Burdzy, and Zhen-Qing Chen, Censored stable processes, Probab. Theory Related Fields 127 (2003), no. 1, 89-152, DOI 10.1007/s00440-003-02751. MR2006232 (2004g:60068)

[10] Luis Caffarelli and Luis Silvestre, An extension problem related to the fractional Laplacian, Comm. Partial Differential Equations 32 (2007), no. 7-9, 1245-1260, DOI 10.1080/03605300600987306. MR2354493(2009k:35096)

[11] Zhen-Qing Chen and Takashi Kumagai, Heat kernel estimates for stable-like processes on dsets, Stochastic Process. Appl. 108 (2003), no. 1, 27-62, DOI 10.1016/S0304-4149(03)00105-4. MR2008600 (2005d:60135)

[12] Rama Cont and Peter Tankov, Financial modelling with jump processes, Chapman \& Hall/CRC Financial Mathematics Series, Chapman \& Hall/CRC, Boca Raton, FL, 2004. MR:2042661 (2004m:91004)

[13] Michael G. Crandall, Hitoshi Ishii, and Pierre-Louis Lions, User's guide to viscosity solutions of second order partial differential equations, Bull. Amer. Math. Soc. (N.S.) 27 (1992), no. 1, 1-67, DOI 10.1090/S0273-0979-1992-00266-5. MR1118699 (92j:35050)

[14] Mark Freidlin, Functional integration and partial differential equations, Annals of Mathematics Studies, vol. 109, Princeton University Press, Princeton, NJ, 1985. MR833742 (87g:60066)

[15] Masatoshi Fukushima, Yōichi Ōshima, and Masayoshi Takeda, Dirichlet forms and symmetric Markov processes, de Gruyter Studies in Mathematics, vol. 19, Walter de Gruyter \& Co., Berlin, 1994. MR1303354(96f:60126)

[16] Maria Giovanna Garroni and Jose Luis Menaldi, Second order elliptic integro-differential problems, Chapman \& Hall/CRC Research Notes in Mathematics, vol. 430, Chapman \& Hall/CRC, Boca Raton, FL, 2002. MR1911531 (2003i:35002)

[17] Qing-Yang Guan, Integration by parts formula for regional fractional Laplacian, Comm. Math. Phys. 266 (2006), no. 2, 289-329, DOI 10.1007/s00220-006-0054-9. MR2238879 (2007j:60074)

[18] Qing-Yang Guan and Zhi-Ming Ma, Reflected symmetric $\alpha$-stable processes and regional fractional Laplacian, Probab. Theory Related Fields 134 (2006), no. 4, 649-694, DOI 10.1007/s00440-005-0438-3. MR2214908(2007a:60034)

[19] Pei Hsu, On excursions of reflecting Brownian motion, Trans. Amer. Math. Soc. 296 (1986), no. 1, 239-264, DOI 10.2307/2000572. MR837810 (87k:60182)

[20] Panki Kim, Weak convergence of censored and reflected stable processes, Stochastic Process. Appl. 116 (2006), no. 12, 1792-1814, DOI 10.1016/j.spa.2006.04.006. MR2307059 (2008b:60004)

[21] José-Luis Menaldi and Maurice Robin, Reflected diffusion processes with jumps, Ann. Probab. 13 (1985), no. 2, 319-341. MR.781408 (86j:60177)

[22] N. Jacob, Pseudo differential operators and Markov processes. Vol. III, Imperial College Press, London, 2005. Markov processes and applications. MR2158336 (2006i:60001) 
[23] P.-L. Lions and A.-S. Sznitman, Stochastic differential equations with reflecting boundary conditions, Comm. Pure Appl. Math. 37 (1984), no. 4, 511-537, DOI 10.1002/cpa.3160370408. MR.745330 (85m:60105)

[24] Pierre-Louis Lions, José-Luis Menaldi, and Alain-Sol Sznitman, Construction de processus de diffusion réfléchis par pénalisation du domaine (French, with English summary), C. R. Acad. Sci. Paris Sér. I Math. 292 (1981), no. 11, 559-562. MR614669 (82f:60172)

[25] S. A. Molčanov and E. Ostrovskiı̌, Symmetric stable processes as traces of degenerate diffusion processes. (Russian, with English summary), Teor. Verojatnost. i Primenen. 14 (1969), 127130. MR0247668(40 \#931)

[26] Luis Silvestre, Regularity of the obstacle problem for a fractional power of the Laplace operator, Comm. Pure Appl. Math. 60 (2007), no. 1, 67-112, DOI 10.1002/cpa.20153. MR2270163 (2008a:35041)

[27] Renming Song and Zoran Vondraček, On the relationship between subordinate killed and killed subordinate processes, Electron. Commun. Probab. 13 (2008), 325-336, DOI 10.1214/ECP.v13-1388. MR2415141 (2009f:60091)

[28] Elias M. Stein, Singular integrals and differentiability properties of functions, Princeton Mathematical Series, No. 30, Princeton University Press, Princeton, N.J., 1970. MR0290095 (44 \#7280)

[29] Daniel W. Stroock, Diffusion processes associated with Lévy generators, Z. Wahrscheinlichkeitstheorie und Verw. Gebiete 32 (1975), no. 3, 209-244. MR0433614 (55 \#6587)

[30] Kazuaki Taira, Boundary value problems for elliptic integro-differential operators, Math. Z. 222 (1996), no. 2, 305-327, DOI 10.1007/PL00004536. MR1429339 (97k:35049)

[31] Kazuaki Taira, Semigroups, boundary value problems and Markov processes, Springer Monographs in Mathematics, Springer-Verlag, Berlin, 2004. MR2019537 (2004i:47080)

Laboratoire de Mathématiques et Physique Théorique, CNRS UMR 7350, Fédération Denis Poisson, FR CNRS 2964, Université François Rabelais, Parc de Grandmont, 37200 TOURs, FRANCE

E-mail address: barles@lmpt.univ-tours.fr

Laboratoire de Mathématiques et Physique Théorique, CNRS UMR 7350, FÉdération Denis Poisson, FR CNRS 2964, Université François Rabelais, Parc de Grandmont, 37200 TOURs, France

E-mail address: Emmanuel.Chasseigne@lmpt.univ-tours.fr

Laboratoire de Mathématiques et Physique Théorique, CNRS UMR 7350, FÉdération Denis Poisson, FR CNRS 2964, Université François Rabelais, Parc de Grandmont, 37200 TOURs, France

E-mail address: christine.georgelin@lmpt.univ-tours.fr

Department of Mathematical Sciences, Norwegian University of Science and TechNOLOGY, 7491 TrondHEIM, NORWAY

E-mail address: erj@math.ntnu.no 